\title{
Axisymmetric particle-driven gravity currents
}

\author{
By ROGER T. BONNECAZE ${ }^{1}$ MARK A. HALLWORTH ${ }^{2}$, \\ HERBERT E. HUPPERT ${ }^{2}$ AND JOHN R. LISTER ${ }^{2}$ \\ ${ }^{1}$ Department of Chemical Engineering, The University of Texas at Austin, Austin, \\ TX 78712-1062, USA \\ ${ }^{2}$ Institute of Theoretical Geophysics, Department of Applied Mathematics and Theoretical \\ Physics, University of Cambridge, Silver Street, Cambridge CB3 9EW, UK
}

(Received 12 July 1994 and in revised form 2 November 1994)

Axisymmetric gravity currents that result when a dense suspension intrudes under a lighter ambient fluid are studied theoretically and experimentally. The dynamics of and deposition from currents flowing over a rigid horizontal surface are determined for the release of either a fixed volume or a constant flux of a suspension. The dynamics of the current are assumed to be dominated by inertial and buoyancy forces, while viscous forces are assumed to be negligible. The fluid motion is modelled by the single-layer axisymmetric shallow-water equations, which neglect the effects of the overlying fluid. An advective transport equation models the distribution of particles in the current, and this distribution determines the local buoyancy force in the shallow-water equations. The transport equation is derived on the assumption that the particles are vertically well-mixed by the turbulence in the current, are advected by the mean flow and settle out through a viscous sublayer at the bottom of the current. No adjustable parameters are needed to specify the theoretical model. The coupled equations of the model are solved numerically, and it is predicted that after an early stage both constant-volume and constant-flux, particle-driven gravity currents develop an internal bore which separates a supercritical particle-free region upstream from a subcritical particle-rich region downstream near the head of the current. For the fixed-volume release, an earlier bore is also predicted to occur very shortly after the initial collapse of the current. This bore transports suspended particles away from the origin, which results in a maximum in the predicted deposition away from the centre.

To test the model several laboratory experiments were performed to determine both the radius of an axisymmetric particle-driven gravity current as a function of time and its deposition pattern for a variety of initial particle concentrations, particle sizes, volumes and flow rates. For the release of a fixed volume and of a constant flux of suspension, the comparisons between the experimental results and the theoretical predictions are fairly good. However, for the current of fixed volume, we did not observe the bore predicted to occur shortly after the collapse of the current or the resulting maximum in deposition downstream of the origin. This is unlike the previous study of Bonnecaze et al. (1993) on two-dimensional currents, in which a strong bore was observed during the slumping phase. The radial extent $R$ of the deposit from a fixed-volume current is accurately predicted by the model, and for currents whose particles settle sufficiently slowly, we find that $R=1.9\left(g_{0}^{\prime} V^{3} / v_{s}^{2}\right)^{1 / 8}$, where $V$ is the volume of the current, $v_{s}$ is the settling velocity of a particle in the suspension and $\mathrm{g}_{0}^{\prime}$ is the initial reduced gravity of the suspension. 


\section{Introduction}

Particle-driven gravity currents are suspensions of dense particles that intrude laterally into an ambient fluid due to the difference between the bulk density of the suspension and that of the ambient fluid. Since the buoyancy force depends on the concentration of particles, there is a strong coupling between the sediment transport and the dynamics of the gravity current. The behaviour of particle-driven gravity currents has application to many geological, oceanographic and environmental situations. These currents, often called turbidity currents, play a major role in the formation of submarine canyons and the transport of silt to the abyssal plains of the deep oceans (Inman, Nordstrom \& Flick 1976). In some cases the sedimentary deposits from ancient turbidity currents have become valuable oil reservoirs (Perrodon 1985). The effects of potentially damaging turbidity currents are often a consideration in the design of reliable submarine structures, such as cables and pipelines (Simpson 1987). In the form of industrial effluents, particle-driven gravity currents are an important means for the transport of pollutant-laden sediment. For example, ash from coal-fired power plants or tailings from mines located near a coast have been dumped into inlets producing turbidity currents (Normark \& Dickson 1976; Hay 1987). The fate of these particles, which often contain toxic metals, is of significant environmental concern.

In a previous paper (Bonnecaze, Huppert \& Lister 1993, hereafter referred to as $\mathrm{BHL}$ ) we studied the dynamics and deposition of two-dimensional particle-driven gravity currents produced by the instantaneous release of a fixed volume of a dense suspension. Here we consider axisymmetric currents for both fixed volumes and fixed fluxes, though some of the salient features of BHL need to be reviewed so that the present paper can be understood on its own. We developed a model in which the dynamics of the current are controlled by a balance between the inertial forces of the moving fluid and the buoyancy forces derived from the suspension of dense particles. In the model the flow of the current is described by the shallow-water equations, which are derived from the usual hydraulic assumptions of vertically uniform flow and a hydrostatic pressure distribution within the current, modified to include the contribution of the particles to the buoyancy force. Gain or loss of fluid at the top of the current is taken to be negligible. The particles are transported within the current by advection, and they are assumed to settle out through a viscous sub-layer at the bottom without re-entrainment. At any position along the current the concentration of particles is assumed to be vertically uniform due to turbulent mixing. The head of the current is modelled as a sharp moving front where a Froude-number relationship is applied between the velocity at the front and its height (Benjamin 1968; Huppert \& Simpson 1980).

The experimental results in BHL for two-dimensional particle-driven gravity currents showed that when the depth of the current was comparable to the depth of the ambient fluid, the dynamics of the fluid overlying the current must also be included in the model. The same hydraulic assumptions were applied, and the so-called two-layer model includes the conservation of mass and momentum of the overlying fluid, which is coupled to the flow of the current. The model is only slightly more complicated, but the improvement in comparisons between the theoretical predictions and our experimental results was substantial.

The numerical solution of these model equations showed that two types of travelling shocks can occur in such particle-driven gravity currents. An internal travelling shock occurs in the later stages of the propagation, whether computed with the single-layer 
or the two-layer model. The shock separates a particle-free, jet-like flow in the rear of the current from a dense, buoyancy-driven flow near the front. The other type of bore is observed to occur only during the initial collapse or slumping phase of currents that have an initial height comparable to the depth of the surrounding fluid. The bore, which is captured numerically only by the two-layer model, is formed when overlying fluid moving in to replace the slumping current reflects from the endwall. The bore occurs irrespective of whether the current is homogeneous or particle-driven, but it strongly affects the particle transport and the dynamics of the current for particledriven flows. It sweeps particle-laden fluid away from the endwall, which results in a slight maximum in the particle deposition away from the wall and a greater depth of deposit downstream compared to that predicted by the single-layer model for a current in a deep ambient fluid. Indeed it is the more important shock for modelling the experiments and is critical for the accurate prediction of the deposition patterns of the sediment.

The agreement between the theoretical predictions using the two-layer model and the experimental measurements for the lengths of particle-driven currents as functions of time and their deposition patterns was very good. The agreement is especially significant because the model has no adjustable parameters. The Froude-number condition at the nose was determined from previous independent experiments on homogeneous gravity currents, and the settling velocity was just that determined from theoretical Stokes flow around equivalent spherical particles.

In this paper we apply the method developed in BHL to axisymmetric gravity currents produced by the release of either a fixed volume or a constant flux of a dense suspension. In $\$ 2$ we describe the details of our model for radially spreading, particle-driven gravity currents. As for a two-dimensional current, the model assumes that the dynamics of the current are dominated by a balance between gravitational and inertial forces and that the flow is sufficiently turbulent for the particles to be well mixed. The axisymmetric shallow-water equations account for the conservation of mass and momentum of the fluid, with the gravitational force depending on the concentration of particles. A vertically averaged advective conservation equation, which includes the effects of settling, accounts for the transport of the particles. A Froude-number condition is required at the front, which relates the velocity, height and reduced buoyancy at the front. As in the two-dimensional case, the model contains no adjustable parameters.

In $\$ 3$ we discuss the behaviour of axisymmetric particle-driven gravity currents created by the release of a fixed volume of fluid. From the numerical solution of the model equations, we present typical height, velocity and concentration profiles. In particular, we discuss how some knowledge of the structure of the current due to the internal shocks, or bores, is necessary for an accurate prediction of the deposition pattern. We also present a simple relationship for the length of the deposit based on scaling analysis and numerical simulations.

We then describe some laboratory experiments in which a fixed volume of suspension was released in a radial sector tank. Experimental measurements of the radial position of the current as a function of time and the sedimentation pattern are then compared to the numerical predictions of the model. It is rather surprising that for an axisymmetric particle-driven gravity current, the single-layer shallow-water equations with a modified nose condition are sufficient to predict our experimental measurements. This is unlike the previous work by BHL on two-dimensional currents, in which, as mentioned earlier, it was necessary to include the effects of the relatively shallow overlying ambient fluid in the theoretical model to simulate the 
bore that occurs during the slumping phase. While this bore would be predicted to be even stronger and its effect on the deposition more dramatic using the axisymmetric two-layer shallow-water equations, experimentally it is not observed to occur.

In $\$ 4$ similar numerical and experimental results are presented for axisymmetric particle-driven gravity currents created by the release of a constant flux of suspension. Finally, in $\$ 5$ we summarize our results and conclude with a discussion of future avenues of research into the behaviour of particle-driven gravity currents. An appendix presents the analytic results from a simple box model with uniform properties in both the horizontal and vertical directions, against which our more rigorous numerical results can be compared.

\section{Theory}

\subsection{Model}

We consider an axisymmetric particle-driven gravity current of bulk density $\rho_{c}(r, t)$, where $r$ is the radial position and $t$ is time, spreading over a horizontal surface into a deep body of ambient fluid of lesser density $\rho_{a}$. The current is composed of particles of density $\rho_{p}$ suspended in an interstitial fluid of density $\rho_{i}$. The density of the current is given by

$$
\rho_{c}(\phi)=\left(\rho_{p}-\rho_{i}\right) \phi+\rho_{i},
$$

where $\phi(r, t)$ is the volume fraction of particles in the suspension.

The gravity current considered here is produced by the release of either a fixed volume or a constant flux of a well-mixed suspension into a radial sector. The flow is axisymmetric within the angle $\theta$ of the sector, which may be up to $2 \pi$. Initially, the Reynolds number of the current is sufficiently large that viscous forces are negligible and the dynamics of the current are dominated by a balance between inertial and buoyancy forces (Huppert 1982). At its inception the current is a complicated threedimensional unsteady flow, but soon after the current has spread sufficiently that its radius $r_{N}(t)$ is very much greater than its slowly varying height $h(r, t)$. Further, the velocity of the current, denoted by $u(r, t)$, is predominantly in the radial direction and there is only a small vertical component. Thus, we may neglect vertical accelerations and assume a hydrostatic pressure distribution within the current, which depends on the local density of the current, $\rho_{c}(\phi)$.

Under these conditions we may describe the current by the radial shallow-water equations, which in dimensionless form are given by

$$
\begin{gathered}
\frac{\partial h}{\partial t}+\frac{1}{r} \frac{\partial}{\partial r}(r u h)=0, \\
\frac{\partial}{\partial t}(u h)+\frac{1}{r} \frac{\partial}{\partial r}\left(r u^{2} h\right)+\frac{\partial}{\partial r}\left(\frac{1}{2}(\phi-\gamma) h^{2}\right)=0,
\end{gathered}
$$

where we have non-dimensionalized lengths, time and velocity by $h_{0},\left(h_{0} / g_{0}^{\prime}\right)^{1 / 2}$ and $\left(g_{0}^{\prime} h_{0}\right)^{1 / 2}$, respectively. Here $h_{0}$ is the characteristic height of the current, which is either the initial height of the current for the instantaneous release of a fixed volume or the height at the inlet for a constant flux of suspension, $g_{0}^{\prime}=\left(\rho_{p}-\rho_{i}\right) \phi_{0} g / \rho_{a}$ is the contribution of the particles to the initial reduced gravity of the current, and $\phi_{0}$ is the initial volume fraction of particles. In equation (3), and throughout the rest of the paper, the volume fraction of particles $\phi$ is rescaled by $\phi_{0}$. We have assumed that $\phi_{0} \ll 1$ and used the Boussinesq approximation, which includes only the effect of $\phi$ on the buoyancy terms and neglects the contribution of the particles to the mass and 
momentum balances. The parameter $\gamma=\left(\rho_{a}-\rho_{i}\right) /\left[\left(\rho_{p}-\rho_{i}\right) \phi_{0}\right]$ is the contribution of the interstitial fluid to the reduced gravity. In this paper we only consider cases in which the densities of the interstitial and ambient fluid are equal, so that $\gamma=0$. This is in contrast to an earlier study on two-dimensional currents (Sparks et al. 1993), which also considered positive values of $\gamma$.

The hydrostatic pressure distribution and driving buoyancy force in the current depend on the local volume fraction of particles. The concentration of particles varies throughout the current due to advection and settling. Here we neglect particle entrainment from the base on the assumption that the current velocities are insufficient to lift deposited sediment into the current. We consider the flow to be sufficiently vigorous, however, that turbulent mixing maintains a vertically uniform particle concentration in the current, without any release of particle-free fluid at the top of the current. We assume that the particles leave the current only through the viscous sublayer at the base with a flux $v_{s} \phi$, where $v_{s}$ denotes the settling velocity of an isolated particle, which is appropriate when the particle concentration is small. This model of particle settling has been used successfully in the study of two-dimensional particle-driven gravity currents (BHL; Sparks et al. 1993) and in earlier studies of sedimentation from turbulent suspensions (Einstein 1968; McCave 1970; Martin \& Nokes 1988, 1989). The dimensionless equation for the conservation of particles is then given by

$$
\frac{\partial \phi}{\partial t}+u \frac{\partial \phi}{\partial r}=-\beta \frac{\phi}{h}
$$

where the settling number $\beta=v_{s} /\left(g_{0}^{\prime} h_{0}\right)^{1 / 2}$ is the dimensionless settling velocity.

Various boundary conditions are necessary to complete our mathematical description of the current. Von Kármán (1940) and Benjamin (1968) showed that the velocity of the nose of the current $u_{N}$ is proportional to the local shallow-water wave speed. Huppert \& Simpson (1980) determined experimentally the proportionality constant for gravity currents created by the release of a fixed volume. Their results can be expressed as

$$
u_{N}=\operatorname{Fr}\left[\left(\phi_{N}-\gamma\right) h_{N}\right]^{1 / 2},
$$

for a particle-driven gravity current, where the Froude number $F r$ is given by

$$
\begin{aligned}
F r & =1.19 & & \left(0 \leqslant h_{N} / H \leqslant 0.075\right) \\
& =0.5\left(h_{N} / H\right)^{-1 / 3} & & \left(0.075 \leqslant h_{N} / H \leqslant 1\right),
\end{aligned}
$$

and $h_{N}$ and $\phi_{N}$ are the height and volume fraction of the current at the front and $H$ is the depth of the ambient fluid. For a gravity current produced by a constant flux of dense fluid with $h_{N} / H \ll 1$, Huppert \& Simpson (unpublished data, 1980) found that

$$
u_{N}=0.72\left[\left(\phi_{N}-\gamma\right) h_{N}\right]^{1 / 2} \text {, }
$$

so that $F r=0.72$ in this case. This so-called Froude condition is a convenient means of parameterizing the unsteady three-dimensional motion at the head of the current at a consistent level of description with the shallow-water equations. As a result of this parameterization, the velocity and height profiles in the shallow-water equations end abruptly at the head. For a constant-flux current, fluid continually flows into the bottom of the head from the body of the curent and is shed from the top, which is unlike the case for a current produced by the release of a fixed volume. These different dynamics are thought to be the cause of the differing Froude conditions at 
the front of currents of constant flux and fixed volume. Note that the experimentally determined Froude numbers include the effects of viscous drag and Reynolds stresses along the head of the current.

For an axisymmetric release of a fixed volume of a suspension, the second boundary condition is that the velocity vanishes on a cylindrical wall at $r=r_{0}$, or

$$
u\left(r_{0}, t\right)=0 \text {, }
$$

where $r_{0}$ may be zero. For an axisymmetric gravity current created by a constant input of suspension into a sector of angle $\theta$ at $r=r_{0}$, where $0<\theta \leqslant 2 \pi$, the flux $Q$ is specified by

$$
r_{0} \theta u\left(r_{0}, t\right) h\left(r_{0}, t\right)=Q .
$$

Because equations (2)-(4) are hyperbolic in character, the number of boundary conditions required to obtain a unique solution is equal to the number of characteristics that propagate from the boundaries into the flow domain. If the flow at the source is either critical or supercritical then there are two inwardly propagating characteristics (BHL), and we must specify the height at the inlet,

$$
h\left(r_{0}, t\right)=1 \text {, }
$$

by our assumed non-dimensionalizations. For subcritical inlet conditions, or for the release of a fixed volume of suspension, equations (5) and (7a) are sufficient. Finally, the initial radius and height of the current must also be specified.

\subsection{Similarity solutions for $\beta=0$}

Suppose that the total volume of the radial current is given by $A t^{\alpha}$. With this supposition currents of fixed volume and constant flux correspond to $\alpha=0$ and 1 , respectively. When $\beta=0$ and there is no settling, equations (2) - (7) may possess long-time similarity solutions (Hoult 1972; Chen 1980) of the form

$$
\begin{aligned}
& h(x, t)=\dot{r}_{N}^{2}(t) H(y), \\
& u(x, t)=\dot{r}_{N}(t) U(y),
\end{aligned}
$$

where

$$
\begin{gathered}
r_{N}(t)=C\left(\frac{g^{\prime} A}{h_{0}^{4} \theta}\right)^{1 / 4}\left(\frac{h_{0}^{4}}{g^{\prime}}\right)^{(\alpha+2) / 4} t^{(\alpha+2) / 4}, \\
C=\left[\left(\frac{\alpha+2}{4}\right)^{2} \int_{0}^{1} y H(y) \mathrm{d} y\right]^{-1 / 4},
\end{gathered}
$$

and the similarity variable $y=r / r_{N}(t)$. Substituting equations (8) and (9) into (2) and (3), we obtain the coupled nonlinear ordinary differential equations

$$
\begin{gathered}
y H U^{\prime}+y(U-y) H^{\prime}+[U+2(\alpha-2) /(\alpha+2) y] H=0, \\
(U-y) U^{\prime}+H^{\prime}+(\alpha-2) /(\alpha+2) U=0 .
\end{gathered}
$$

For a current of fixed volume $(\alpha=0)$ in deep surroundings the self-similar height and velocity are given by

and

$$
H(y)=\frac{1}{2}\left(y^{2}-1\right)+F r^{-2}
$$

$$
U(y)=y,
$$



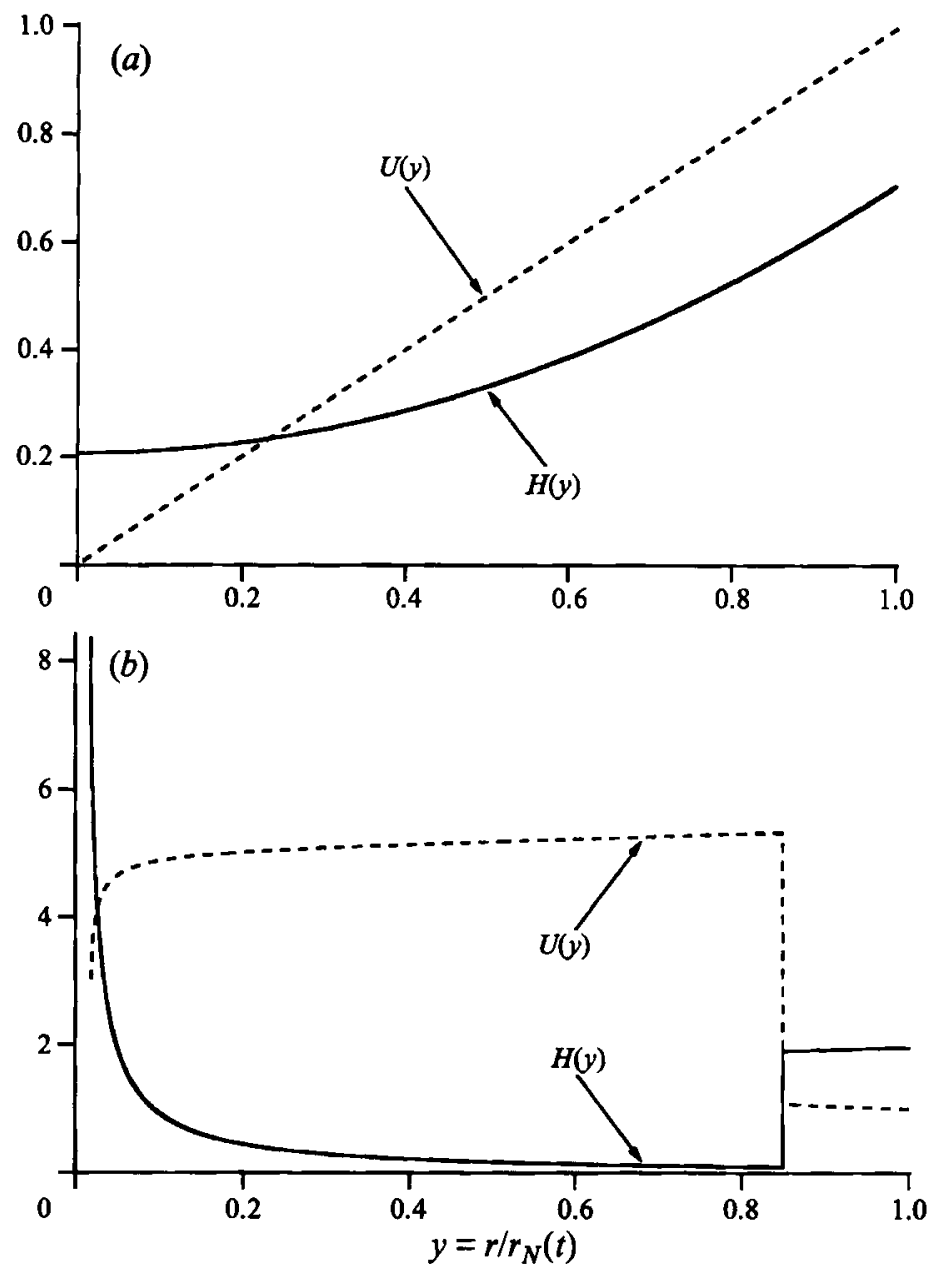

FIGURE 1. The self-similar velocity $U(y)$ and height $H(y)$ for a homogeneous gravity current created by the release of $(a)$ a fixed volume with $F r=1.19$ at the nose and $(b)$ a constant flux with $F r=$ 0.72 . For the latter case, the shock is assumed to be located at $y_{s}=0.85$.

which are illustrated in figure $1(a)$ for $F r=1.19$. In this case $C=1.29$. The velocity increases linearly from zero at the tail to the velocity of the nose at the head, and the height of the current increases quadratically from the tail to the head. The variation in the height of the current creates an adverse pressure gradient, which decelerates the flow. This loss of momentum throughout the current may be attributed to the nose condition, which represents a sink of momentum. This is qualitatively identical to the behaviour of two-dimensional homogeneous currents.

For radial currents where $\alpha>0$, a continuous similarity solution on $y \in[0,1]$ does not exist, as was shown by Grundy \& Rottman (1986). However, since the shallow-water equations are hyperbolic, Grundy \& Rottman suggested, though never showed, that self-similar solutions with shocks may exist. This is in fact the case for a radial current produced by a constant flux $(\alpha=1)$. For a weak solution to be self-similar, the shock speed must be given by $U_{s}=y_{s} r_{N}(t)$, where $y_{s}$ is the location of the shock in similarity space. The location of the shock depends on the details of 
the flow near $r=0$. Such shocks also occur for two-dimensional currents and have been considered in detail by Gratton \& Vigo (1994).

Figure $1(b)$ illustrates typical profiles of the self-similar velocity and height for a constant-flux radial current, assuming $F r=0.72$ at the front and, as an example, $y_{s}=0.85$. To construct these profiles, equation (12) was integrated backwards to $y_{s}$ with the boundary condition $U(1)=1$ and $H(1)=F^{-2}$. The conjugate states were then found at $y_{s}$ using the shallow-water shock equations (e.g. Whitham 1974), and the backward integration was continued to near $y=0$. For this particular profile $C=0.96$. The location of the shock in similarity space depends on the inlet conditions, as discussed by Grundy \& Rottman (1986).

The profiles for different inlet conditions are qualitatively similar. The velocity increases rapidly near the inlet and then more gradually until the shock, where it changes discontinuously to a lesser value. The velocity then decreases only very slightly to the head of the current. The height of the current decreases rapidly at first near the inlet of the current and then much less rapidly until the shock, where it jumps to a greater value that increases slightly to the head.

The formation of the shock can be explained by considering the Froude condition at the nose and conservation of fluid. Away from the inlet, the velocity is nearly constant and the height decreases as $y^{-1}$ or $r^{-1}$ such that $r \theta u h=Q$ is constant. Since the height is small, the local Froude number, $u h^{-1 / 2}$, is large and the flow is supercritical. The flow at the head, however, is subcritical due to the imposed condition $F r=0.72$ at the front. In order to match these flows, a shock or bore must be formed.

As was observed in BHL and as we shall see here, the loss of particles and the resulting changes in the local density of the current markedly change the shape of the profiles from those in figure 1. In particular, it is found from numerical solution of (2)-(7) that additional shocks develop in a fixed-volume current with $\beta \neq 0$ and that the magnitudes of the shocks in a constant-flux current are larger. However, at intermediate times the general structure of the computed radial particle-driven gravity currents for $\beta \neq 0$ resembles that of the similarity profiles for $\beta=0$. The numerical method used to obtain these results is a simple adaptation to cylindrical coordinates of the method described in BHL, to which we refer the interested reader for details.

\section{Currents of fixed volume}

\subsection{Numerical profiles}

The height, velocity and concentration profiles for a radial gravity current created by an instantaneous fixed-volume release are presented in figures 2 and 3. Initially, the fixed volume of fluid is at rest and its radius is twice the starting height. For these profiles, the settling number $\beta=5 \times 10^{-3}$, which is in the range of our experimental values.

As was the case for two-dimensional particle-driven gravity currents, the dynamics of radial particle-driven gravity currents can be approximately divided into three phases: an initial phase, during which the fixed volume of fluid collapses, forms a weak internal shock and very few particles have settled; a transition phase, in which particles are preferentially removed from the rear of the current and the fluid in the tail decelerates less rapidly than the nose; and a travelling shock phase, during which a bore develops within the current and separates a particle-free jet-like region from a relatively particle-rich gravity current region. 

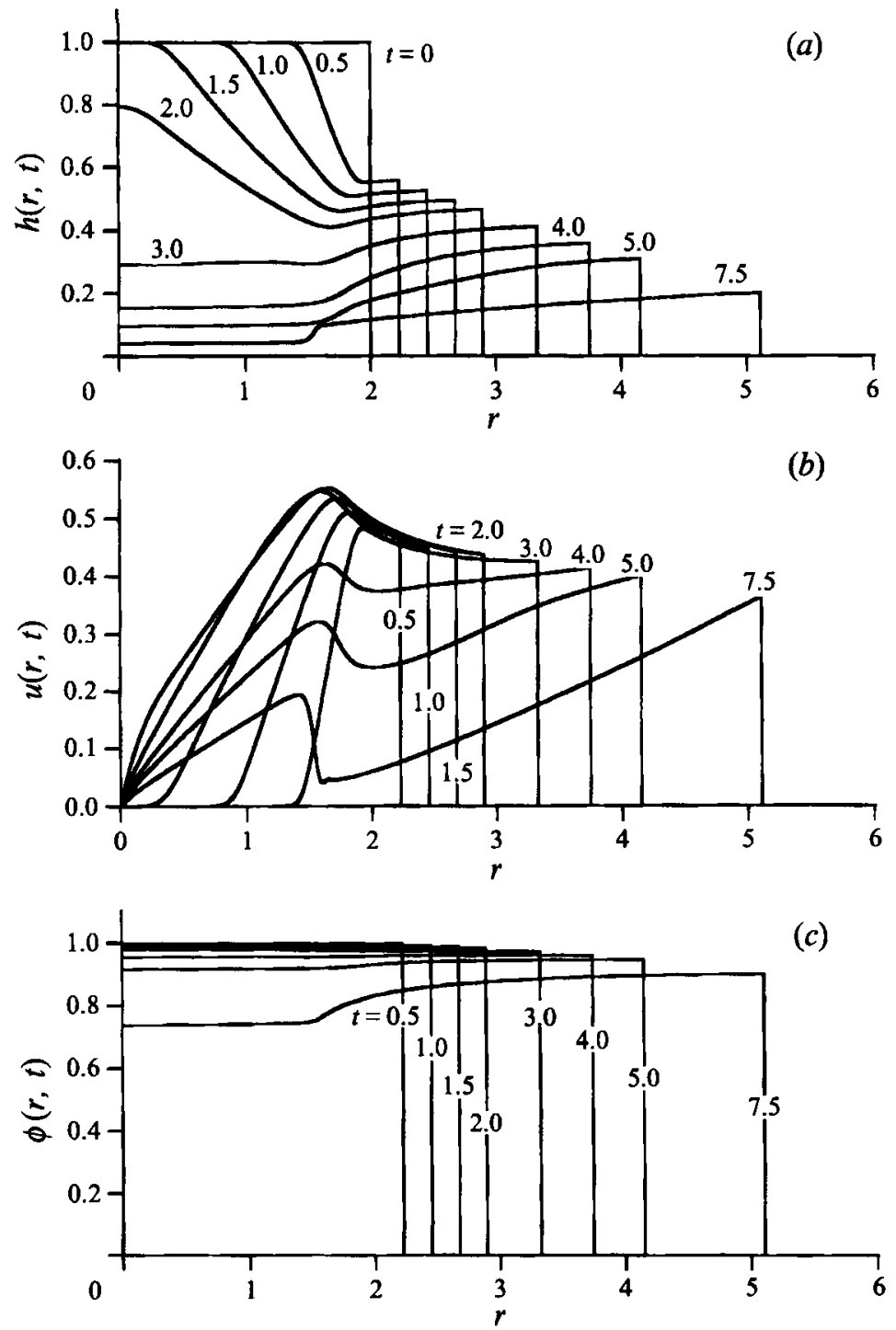

FIGURE 2. The numerically determined profiles of $(a)$ height $h(r, t),(b)$ velocity $u(r, t)$ and $(c)$ concentration $\phi(r, t)$ at early times of a collapsing particle-driven gravity current of fixed volume. The current starts from rest with dimensionless height $h_{i}=1$ and radius $r_{N}(0)=2$. The settling number $\beta=5 \times 10^{-3}$. The successive non-dimensional times are noted by each profile.

Figure 2 illustrates the profiles during the initial phase. The fluid initially collapses from the front of the current and creates a wave that travels backwards towards the origin. This wave is eventually reflected toward the front, ultimately becoming a weak shock as seen at $t=7.5$. The formation of this shock is most apparent in the velocity profiles depicted in figure $2(b)$. However, by the time the shock becomes pronounced, the conditions on either side of it are such that the shock is moving backwards, as though it were reflected off the forward section of the current. The shock travels backwards and at the origin of the current is again reflected between $t=7.5$ and 20.0 (figure 3). It then travels forward in the form of a small wave, which moves more slowly than the nose of the current and thus never reaches the front. 

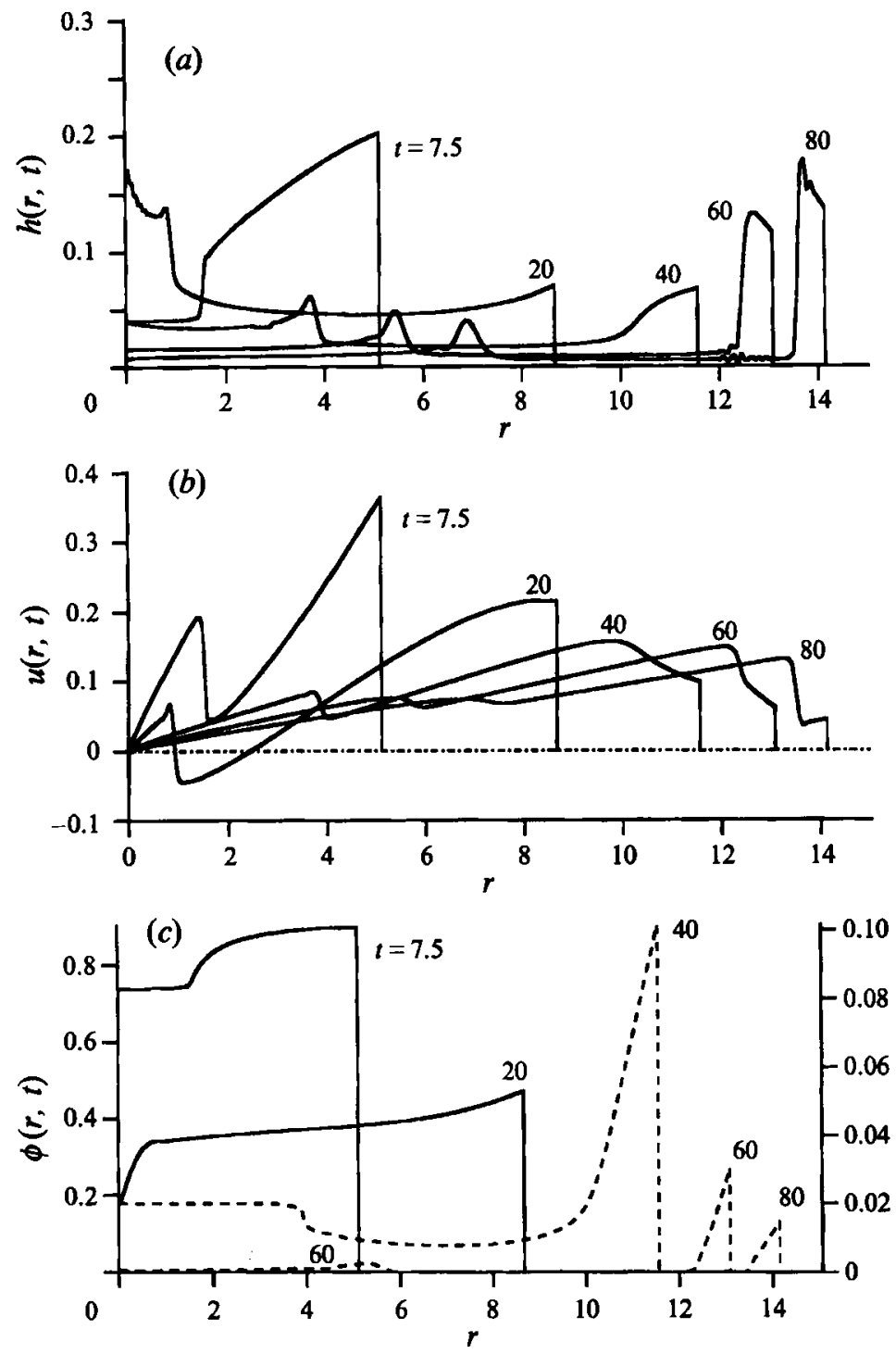

FIGURE 3. The numerically determined profiles at later times for the current described in figure 2. Note that the ordinate on the right is used for the volume fraction (dashed curves) at later times.

This initial sequence of events is also observed for a homogeneous current with no settling, though eventually the velocity and height in that case attain their self-similar profiles.

As particles settle out of the current, however, the structure changes markedly from that of a homogeneous current. After about $t=20$ in figure 3 the fluid at the rear of the current no longer decelerates as rapidly as the nose and the maximum velocity occurs in the body of the current. During this transition phase, the height of the current near the front increases and the concentration of particles in the rear of the current decreases rapidly. Finally, a travelling shock forms in the current for $t>40$, which separates a particle-free jet-like region in the rear from a relatively particle-rich region near the front. Note that the oscillations and slight overshoot for the height 
of the current near the shock are due to the limitations of the numerical method, but generally do not influence the results, as we confirmed by varying the grid size, time step and dissipation parameter.

The formation of the first shock after the collapse of the current is unlike the behaviour of two-dimensional currents modelled with the single-layer shallow-water equations, where only the travelling shock in the later stages of the current is observed. This initial shock is due to the axisymmetric nature of the flow. Because of the diverging character of the flow, the radius of the current increases less rapidly than the radial position of the shock or wave. This wave pushes fluid towards the front, and eventually the height and velocity on the downstream side of the shock front are such that the wave is reflected backwards, much like a wave reflecting off a moving wall. This does not occur for two-dimensional currents because the front of the current travels faster than the wave, which results in the wave being stretched and disappearing into the current.

The formation of the subsequent travelling shock is due to differential settling of particles along the length of the current, as in the case of two-dimensional particledriven gravity currents. We recall that the variation in height from the head to the tail of the current establishes a pressure gradient that decelerates the fluid behind the nose. As shown by equation (4), the volume fraction of particles decreases more rapidly at the rear of the current, where the height is small. Consequently, the density and pressure gradient in the tail are reduced, and the fluid there is not decelerated as rapidly as at the nose. The difference in velocities becomes so great that a shock develops to match the particle-free jet-like region to the relatively particle-rich nearhead region. We see from figures $2(c)$ and $3(c)$ that the differential settling is apparent at around $t=7.5$ and its effect becomes significant between $t=20$ and 40 .

\subsection{Experimental results}

Several experiments were performed to determine the radius of a fixed-volume gravity current as a function of time and its resulting deposition pattern for comparison with our numerical model. The experiments were performed in a radial sector tank of half-angle $4^{\circ}$ made of clear Perspex, as depicted in figure $4(a)$. The tank was $20 \mathrm{~cm}$ high and about $210 \mathrm{~cm}$ long. A rectangular lock $30.6 \mathrm{~cm}$ long and $3.8 \mathrm{~cm}$ wide was attached to the tank instead of a sharp apex. This allowed us to mix the suspension easily and prevent sedimentation in a sharp corner. The tank was filled with tap water to a depth of $14 \mathrm{~cm}$, and a Perspex gate with foam seals around its edges was placed at either 15 or $30 \mathrm{~cm}$ from the endwall of the rectangular lock.

The suspension for the current was made by mixing fairly monodisperse, noncohesive silicon carbide particles $\left(\rho_{p}=3.217 \mathrm{~g} \mathrm{~cm}^{-3}\right)$. As a precaution, a small amount of Calgon was added to prevent any coagulation of the particles. Two combinations of particle diameter and lock length are presented: $23 \mu \mathrm{m}$-diameter particles $\left(v_{s}=0.064 \mathrm{~cm} \mathrm{~s}^{-1}\right)$ in a $30 \mathrm{~cm}$ long lock and $37 \mu \mathrm{m}$-diameter particles $\left(v_{s}=\right.$ $0.17 \mathrm{~cm} \mathrm{~s}^{-1}$ ) in a $15 \mathrm{~cm}$ long lock. The masses of particles added were such that the initial values of the reduced gravity of the suspensions were 41,21 and $11 \mathrm{~cm} \mathrm{~s}^{-2}$. Under these conditions, $\beta$ ranged from $2.6 \times 10^{-3}$ to $1.4 \times 10^{-2}$.

After the suspension had been well-mixed, the gate was lifted to release the gravity current. As the current propagated down the tank, the position of its front was marked on the clear wall of the tank at $3 \mathrm{~s}$ intervals, and the positions were recorded at the conclusion of the experiment, when all the particles had settled. The tank was divided into annular sections and the sediment in each sector was vacuumed with a siphon tube into a beaker. The water was decanted and the particles dried and 
(a)
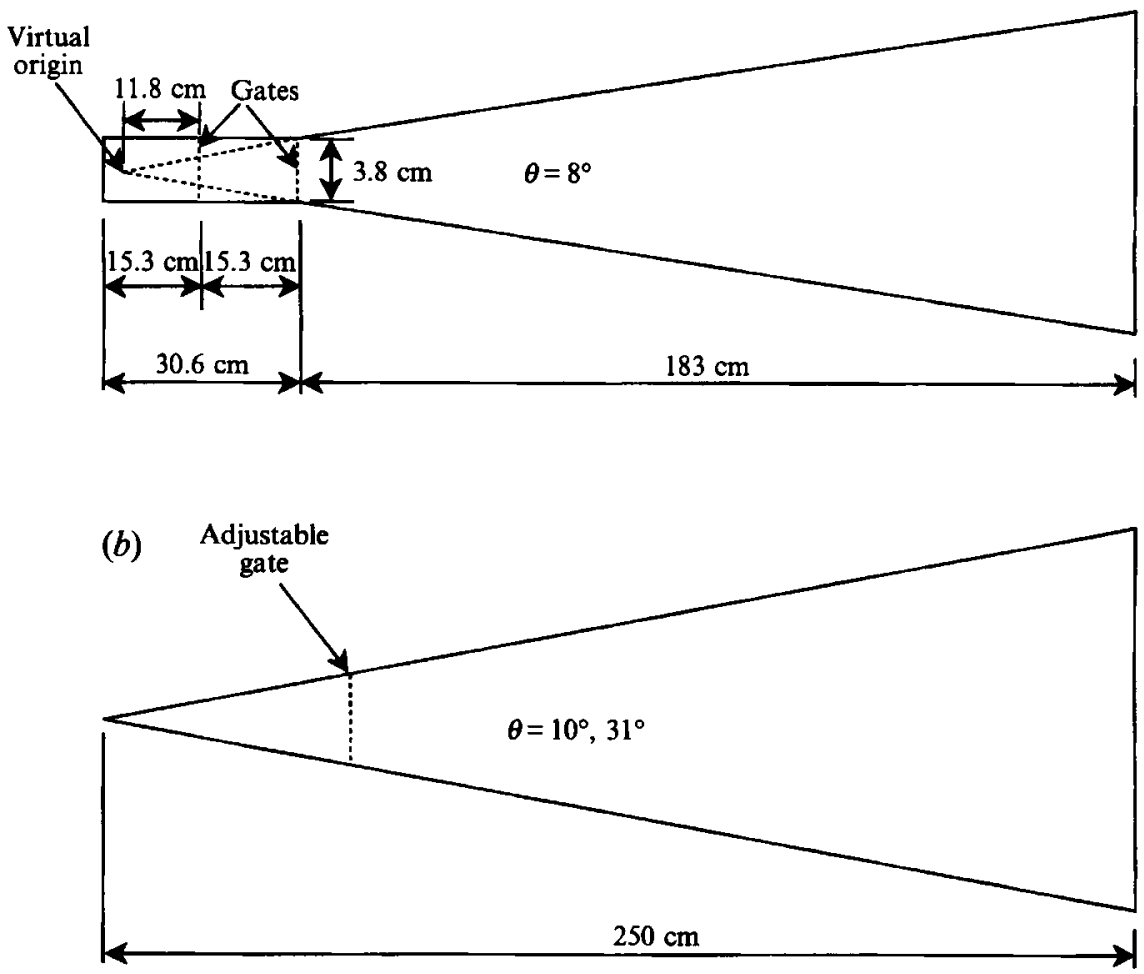

(c)

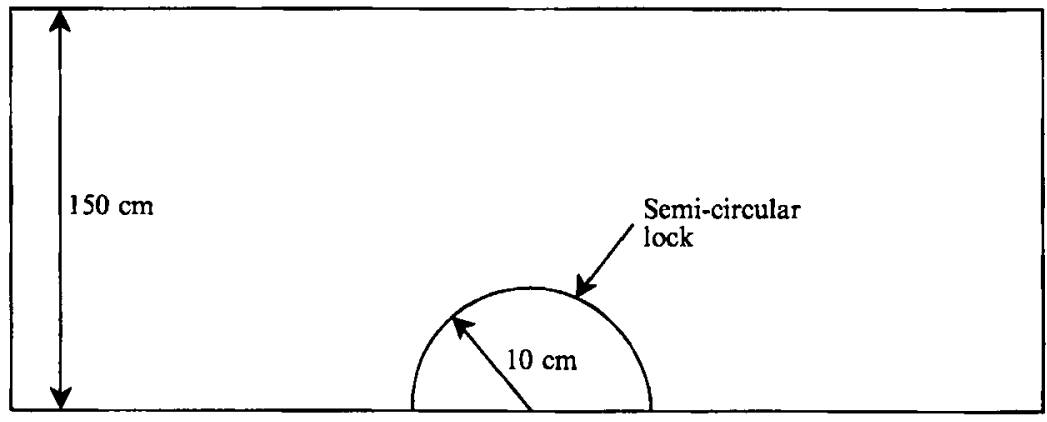

FIGURE 4. Plan views of the tanks used in the experimental studies: (a) sector tank with rectangular lock used for most of the experiments on the release of a constant volume or constant flux of suspension; (b) sector tank with true apex; and (c) large rectangular tank with semi-circular lock for some experiments on the release of a constant volume of suspension.

weighed to determine the mass of deposit per unit area. Comparison with the initial total mass indicatess that these measurements were accurate to within $3 \%$.

Figure 5 presents the radius as a function of time for the axisymmetric particledriven gravity currents. Because the tank had a rectangular lock instead of a sharp apex, radial positions were measured with respect to a virtual origin given by the extended intersection of the tank walls (figure $4 a$ ). At early times, during the socalled slumping phase (Huppert \& Simpson 1980), the radius of the current increased 

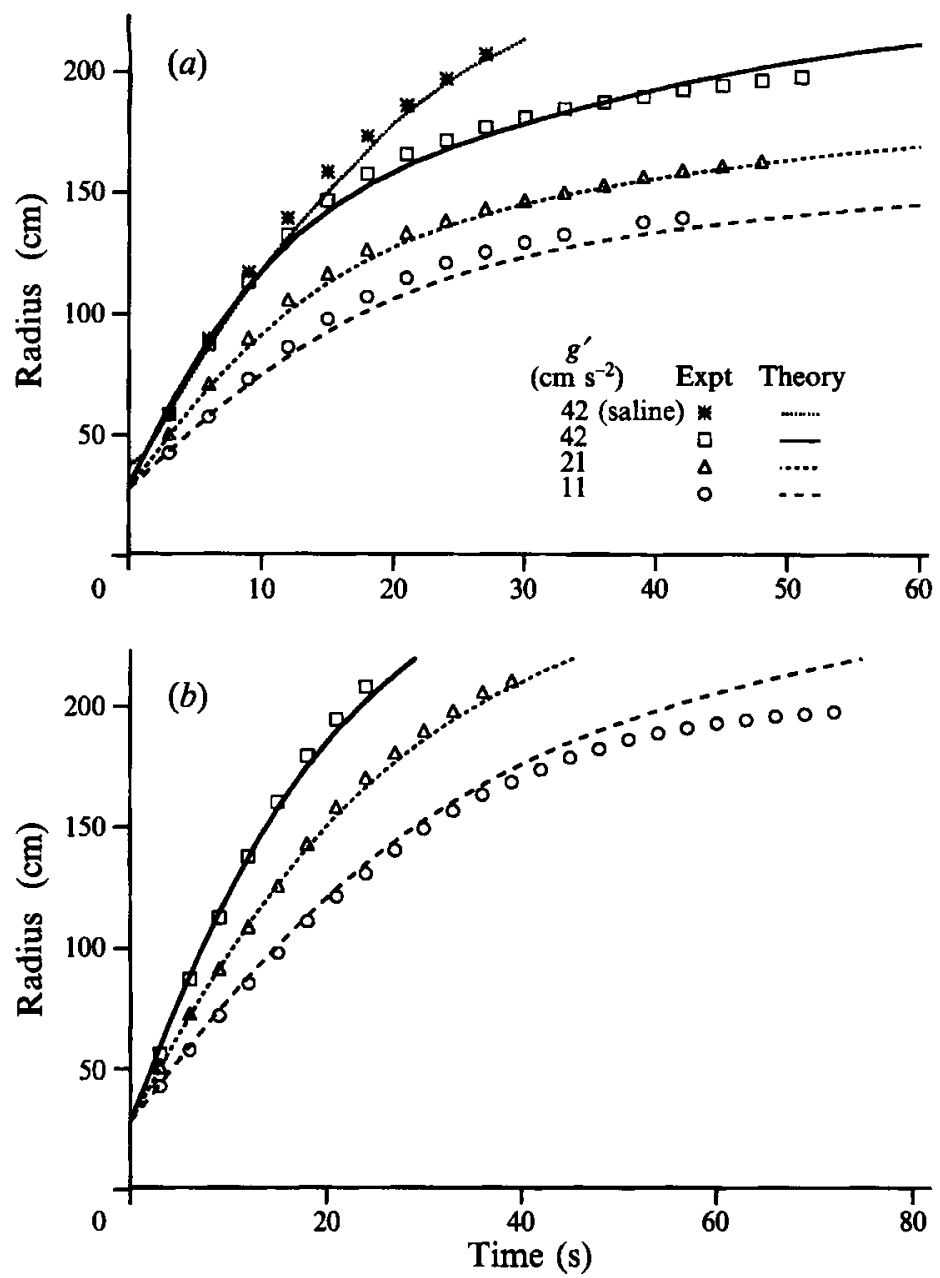

FIGURE 5. The radius as a function of time for axisymmetric gravity currents composed of suspensions of $(a) 37$ and $(b) 23 \mu \mathrm{m}$-diameter silicon carbide particles released into a tank of fresh water. The radial positions were measured with respect to the virtual origin in figure $4(a)$. The symbols are from experimental measurements and the curves are from the numerical solution of the theoretical model. The properties of the current are listed in the legend.

linearly with time, which is captured in the model by the varying Froude number at the front of the current (equation (5)). At later times the radius increased more slowly. As we would expect, the radius of a current at any time increased with increasing initial reduced gravity, since there is a greater buoyancy force driving the flow. Also, currents composed of the smaller $23 \mu \mathrm{m}$-diameter particles travelled further than those composed of the larger $37 \mu \mathrm{m}$-diameter particles, since the latter settled more rapidly and reduced the buoyancy force. In addition to the experimental data, figure 5 presents the numerical prediction of the radius of the currents as a function of time. It is seen that the agreement between the two is excellent. For comparison, the experimental results and numerical prediction for the propagation of a saline, rather than particle-driven, gravity current are also shown in figure $5(a)$. Though the initial rate of propagation is the same as that of the particle-driven current of 

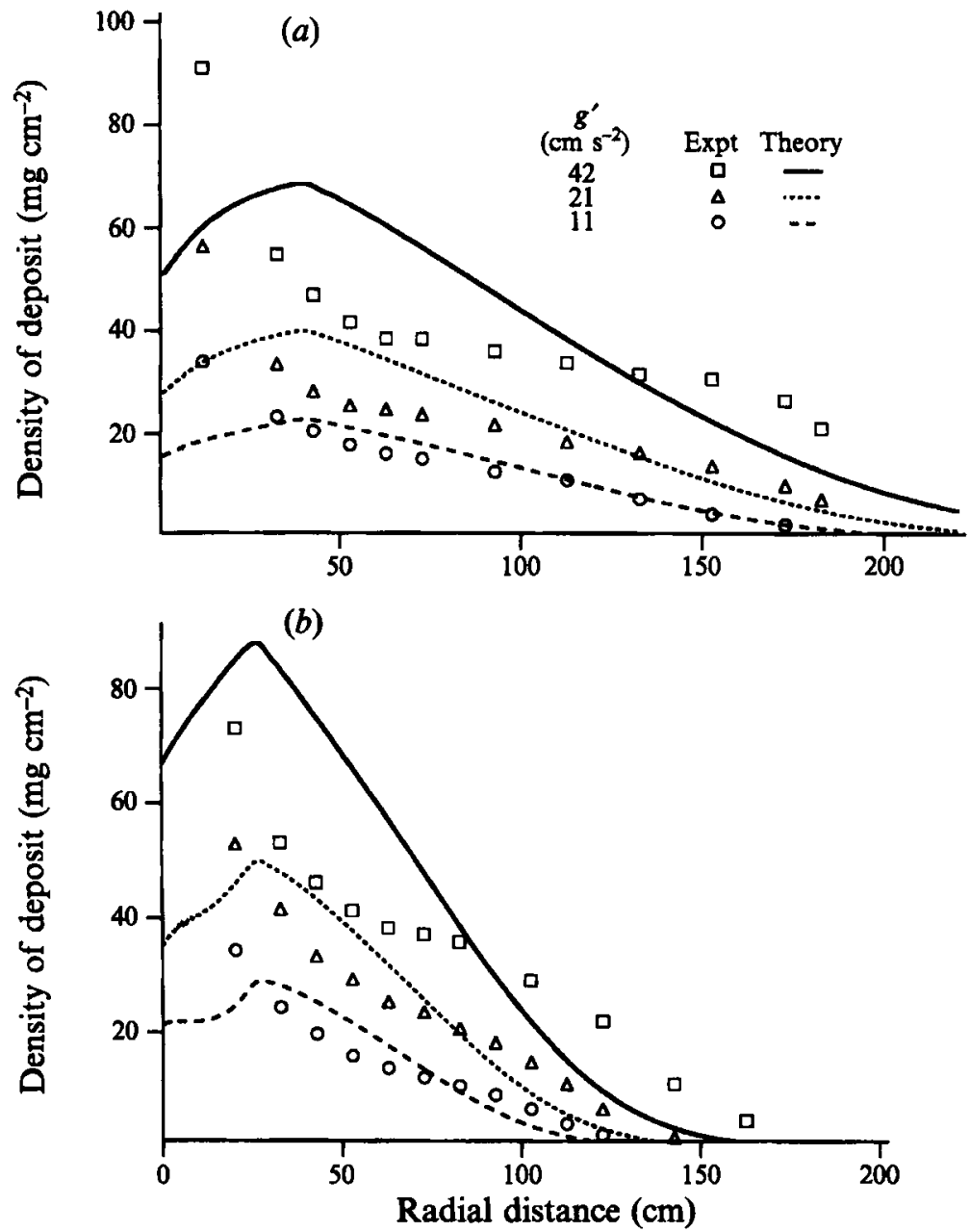

Figure 6. The final areal density of the deposit after the axisymmetric release of a fixed volume of suspension for $(a) 23$ and $(b) 37 \mu \mathrm{m}$-diameter particles. The radial positions were measured with respect to the virtual origin in figure $4(a)$.

equivalent density, the saline current decelerates more slowly since its total buoyancy is conserved.

We also confirmed that these currents were dominated by inertial and not viscous forces. The time $t^{*}$ for transition from an inertially to a viscously dominated homogeneous axisymmetric flow occurs at approximately $t^{*}=0.75\left(Q / \theta g_{0}^{\prime} v\right)^{1 / 3}$, where $v$ is the kinematic viscosity (Huppert \& Simpson 1980). As discussed in BHL, this time of transition is longer for particle-driven currents, so we may use the above formula as a conservative estimate of the transition time. From this estimate we find that all the currents remained in the inertial flow regime for the duration of the experiment with the exception of the current with $23 \mu \mathrm{m}$-diameter particles and $g_{0}^{\prime}=11 \mathrm{~cm} \mathrm{~s}^{-2}$. For this exception, the transition time is estimated to be about $51 \mathrm{~s}$, and indeed the predicted radius as a function of time begins to deviate from the observed value at about that time.

The areal density of the deposits as a function of the downstream radial position is presented in figure 6 for the 23 and $37 \mu \mathrm{m}$-diameter particles. Generally, the 
experimental measurements of the density of a deposit decreased monotonically downstream, dropping most rapidly near the origin. The magnitude of the deposit increased with increasing initial reduced gravity, or equivalently, increasing initial particle loading. The rate of decrease with downstream distance for the density of deposit was less for the smaller diameter particles since they settled out more slowly and were advected further downstream.

For the suspensions composed of $23 \mu \mathrm{m}$-diameter particles the numerical prediction of the density of deposit matches the experimental measurements closely, except near the origin and for the largest value of the initial reduced gravity. The model predicts a slight maximum (figure $6 a$ ) around $50 \mathrm{~cm}$, which was not observed in the experiments. The predicted maximum in the density of deposit occurs because of the initial shock that forms during the collapse of the current. Recall in figure 2(a) that when this shock occurs, particle-laden fluid is swept forward, which leaves relatively fewer particles to settle out near the origin. The trends are qualitatively similar for the suspensions composed of $37 \mu \mathrm{m}$-diameter particles.

Although the agreement between the numerical predictions and the experimental measurements is fairly good, we made several attempts to improve it. Originally, we assumed that the two-layer shallow-water equations would be necessary to account for the overlying fluid, since for some time after release of the suspension the depth of the current and ambient fluid are comparable. Such a modification greatly improved the predictive power of our numerical model for two-dimensional particle-driven gravity currents. Compared to the single-layer model, however, the axisymmetric two-layer model predicts a greater maximum in the density of deposit which is located even further downstream. A stronger shock occurs on collapse in the two-layer model because of the counterflow of the ambient fluid (BHL). This sweeps more fluid further downstream and leaves even fewer particles to settle out near the origin. This modification though does improve the agreement between the numerical and experimental deposition patterns far downstream. However, a close examination of a video recording of the collapse of the current in the experiments did not reveal the formation of a strong forward propagating shock as predicted by the two-layer model. The sequence of events depicted in figure 2(a) is much closer to what is observed. Unfortunately, exact details of the structure of the current are necessary to predict accurately the pattern of deposition due to the sensitivity of the rate of settling to the height of the current.

We considered the possibility that the discrepancies might be due to the rectangular lock. To test this hypothesis we conducted experiments in two sector tanks with a sharp apex, as depicted in figure $4(b)$. These tanks were each about $250 \mathrm{~cm}$ long and had half-angles of $5^{\circ}$ and $15.5^{\circ}$. However, experimental measurements of the density of deposit in these tanks were effectively the same as those in the tank with the rectangular lock under equivalent initial conditions. We also considered the additional drag due to the proximity of the sidewalls near the apex, which is not included in the numerical model. To this end an experiment was performed in which a semi-circular cylinder of suspension was released into a large rectangular tank (figure 4c). Again, the density of deposit measured was similar to our initial experiments. Apparently, the sidewalls do not the cause the discrepancies.

What then is their cause? The deposition patterns do not quite match near the endwall since we predict a bore that is not observed. Unfortunately, it is not clear to us why this should be the case in this geometry and not in a two-dimensional flow. One possibility is that the deficit in the mid-range and excess in the tail of the experimental density of deposits compared to the numerical predictions may be due to 
sedimented particles being swept forward along the floor by the flow. The magnitude of this effect should increase with the velocity of the current. Indeed the discrepancy between the measurements and predictions increases with increasing initial reduced gravity and thus the speed and turbulence of the flow, as seen in figure 6.

In summary the comparisons between the numerical predictions and experimental measurements of the radius of the current as a function of time are excellent. The comparisons between the numerical and experimental deposition patterns, while fair, are not as good as for two-dimensional, fixed-volume particle-driven gravity currents. There are no adjustable parameters in the numerical model presented here and, on balance, the model, which predicts the experimental observations fairly well, is useful in its present form.

\subsection{Prediction of the extent of deposition}

The radius of the current as a function of time and the extent of its deposit is wellpredicted by our model (figures 5 and 6). We can thus confidently use the numerical simulations of the model to derive a general quantitative relationship that predicts the extent of the deposit produced by the axisymmetric release of a fixed volume of a suspension.

We start from the observation that when $\beta \ll 1$ we expect the current to propagate many times the initial lock length before sedimentation is complete so that the initial shape of the current is forgotten and its later behaviour depends chiefly on its initial volume $V$. We therefore seek a rescaling of (2)-(7), in terms of the volume and initial reduced gravity of the current and the settling velocity of the particles. Instead of the characteristic dimensions listed in $\$ 2.1$, we non-dimensionalize $r$ by $\beta_{V}^{-1 / 4} V^{1 / 3}, h$ by $h_{V}=\beta_{V}^{1 / 2} V^{1 / 3}, t$ by $h_{V} / v_{s}$ and $u$ by $\left(g_{0}^{\prime} h_{V}\right)^{1 / 2}$, where the modified settling number $\beta_{V}=v_{s} /\left(g_{0}^{\prime 3} V\right)^{1 / 6}$. These scalings are chosen so that the form of (2), (3), (5) and (6) is unchanged, while equation (4) for the conservation of particles, which is now given by

$$
\frac{\partial \phi}{\partial t}+u \frac{\partial \phi}{\partial r}=-\frac{\phi}{h}
$$

no longer depends on $\beta$. The modified settling number $\beta_{V}$ appears only in the new dimensionless initial conditions, which are given by

$$
\begin{gathered}
h(r, 0)=\beta_{V}^{-1 / 2}\left(h_{0} / V^{1 / 3}\right), \\
r_{N}(0)=\beta_{V}^{1 / 4}\left(R_{0} / V^{1 / 3}\right),
\end{gathered}
$$

where $R_{0}$ is the initial dimensional radius of the current.

From this rescaling we may draw two conclusions. First, if $R_{0} V^{-1 / 3} \beta_{V}^{1 / 4}=r_{N}(0) \ll 1$ then the initial dimensionless shape of the current approximates a delta function (corresponding to point release), and the extent of the deposit is independent of the details of the initial conditions. Second, if this condition is satisfied then the scaling of the dimensional extent of the deposit $R$ is given by

$$
R \propto V^{1 / 3} \beta_{V}^{-1 / 4}
$$

Note that these conclusions are true whether the current is released in a shallow or deep ambient fluid, since the modified settling number does not enter into the Froude-number condition imposed at the front of the current. This is to be expected since it is necessary that $\beta_{V} \ll 1$ and/or $h_{0} \gg V^{1 / 3}$ if the extent of the deposit is to be independent of the initial conditions, and for these conditions there will not be much 
settling during the initial collapse of the current. In such cases all currents of volume $V$ and initial reduced gravity $g_{0}^{\prime}$ will behave in the same way regardless of their initial depths relative to the ambient fluid. $\dagger$

Several numerical simulations were performed to determine the constant of proportionality in equation (16) and define the limits of its applicability. Since the extent of deposit never truly vanishes, we choose to define $R$ as the distance at which $99.9 \%$ of the sediment has been deposited. The simulations were performed for currents with varying initial aspect ratios $\left(V / h_{0}^{3}\right.$ ranging from 0.1 to 100$)$ and modified settling numbers $\beta_{V}$ ranging from $10^{-7}$ to $5 \times 10^{-1}$. The initial depth of the current was assumed to be either equal to $\left(h_{0} / H=1\right)$ or very much less than $\left(h_{0} / H \ll 1\right)$ that of the ambient fluid. These two extremes should bracket the extent of the deposit for intermediate values of $h_{0} / H$. Note also that we have assumed here that the current spreads within an angle $2 \pi$ - if the current flows within a sector of angle $\theta$, the volume of the current should merely be replaced by $2 \pi V / \theta$.

Figure 7 $(a)$ presents the dimensionless extent of deposition as a function of the modified settling number. For very small values of $\beta_{V}$ and $R_{0} / h_{0}$, we observe that the scaling in equation (16) is indeed correct and that the extent of the deposit is quantitatively predicted by

$$
R=1.9 V^{1 / 3} \beta_{V}^{-1 / 4} .
$$

The range of applicability of this relationship is best determined from a plot of $R V^{-1 / 3} \beta_{V}^{1 / 4}$ against $R_{0} V^{-1 / 3} \beta_{V}^{1 / 4}$ (figure $7 b$ ). When $R V^{-1 / 3} \beta_{V}^{1 / 4}$ does not vary with $R_{0} V^{-1 / 3} \beta_{V}^{1 / 4}$ the extent of the deposit is predicted by (17). Otherwise, the extent of the deposit depends on the initial height and radius of the current. From figure $7(b)$ we conclude that the extent of deposition is independent of the initial disposition of the current and is predicted by equation (17) when

$$
\begin{aligned}
R_{0} V^{-1 / 3} \beta_{V}^{1 / 4} & \leqslant 0.1 & \text { for } & h_{0} / H=1 \\
& \leqslant 0.3 & \text { for } & h_{0} / H \ll 1 .
\end{aligned}
$$

Finally, we note that when equation (18) is not satisfied, the upper bound for the extent of deposit is defined by (17) and an empirical lower bound is defined by

$$
R=0.92 V^{1 / 3} \beta_{V}^{-1 / 3},
$$

at least for the range of experimental and numerical data presented here.

The constant 1.9 in equation (17) depends of course on the definition of the extent $\boldsymbol{R}$ of deposition as the distance at which $99.9 \%$ of the particles have been deposited or $0.1 \%$ of the particles remain in suspension. The constant was also determined for values up to $10 \%$ of particles remaining in suspension. The results are presented in figure 8, from which we see that the constant increases from 1.2 for $90 \%$ of the particles deposited to 1.9 for $99.9 \%$ of the particles deposited. Though the model predicts that a small proportion of the particles propagate to very large distances, it is clear that the neglected effects of viscous dissipation will eventually halt the weak remnant current and lead to a deposit of finite extent.

$\dagger$ A similar rescaling of the equations in BHL for two-dimensional particle-driven gravity currents shows that $L \propto V^{1 / 2} \beta_{V^{\prime}}^{-2 / 5}$ if $\beta_{V^{\prime}}^{2 / 5}\left(L_{0} / V^{1 / 2}\right) \ll 1$, where $L$ is the extent of the deposit, $L_{0}$ is the initial length of the current, $V^{\prime}$ is the volume per unit width and $\beta_{V^{\prime}}=v_{s} /\left(g_{0}^{\prime 2} V^{\prime}\right)^{1 / 4}$ is the modified settling number. This relationship has been verified experimentally (Dade \& Huppert 1994) and the constant of proportionality determined to be 3 . 


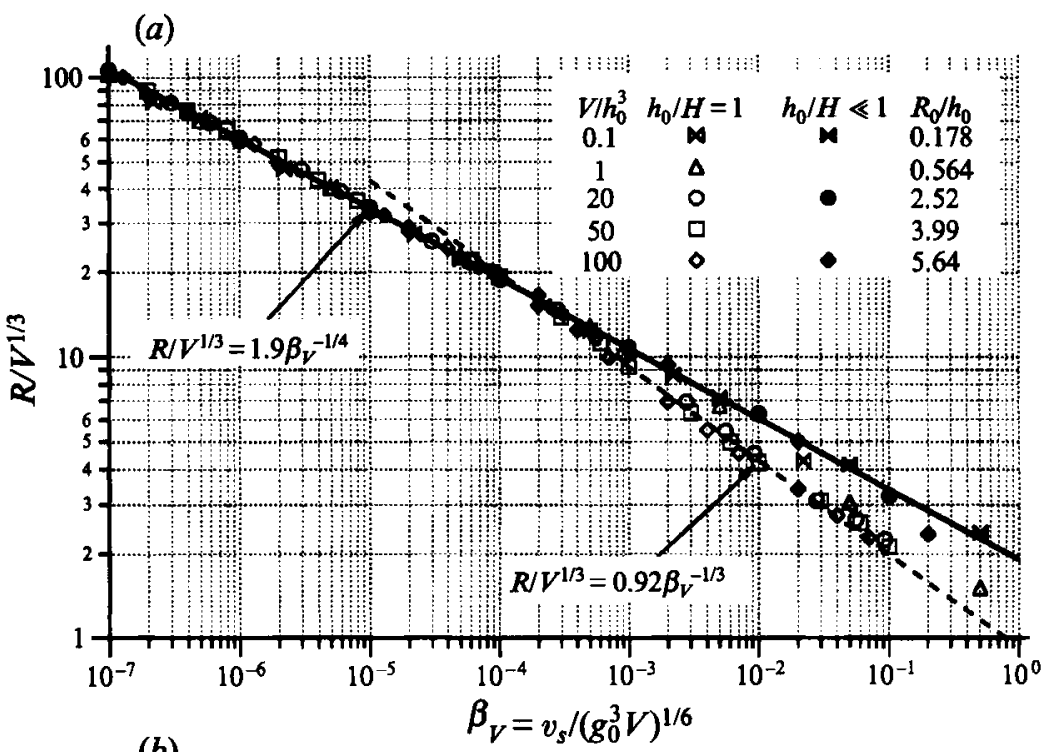

(b)

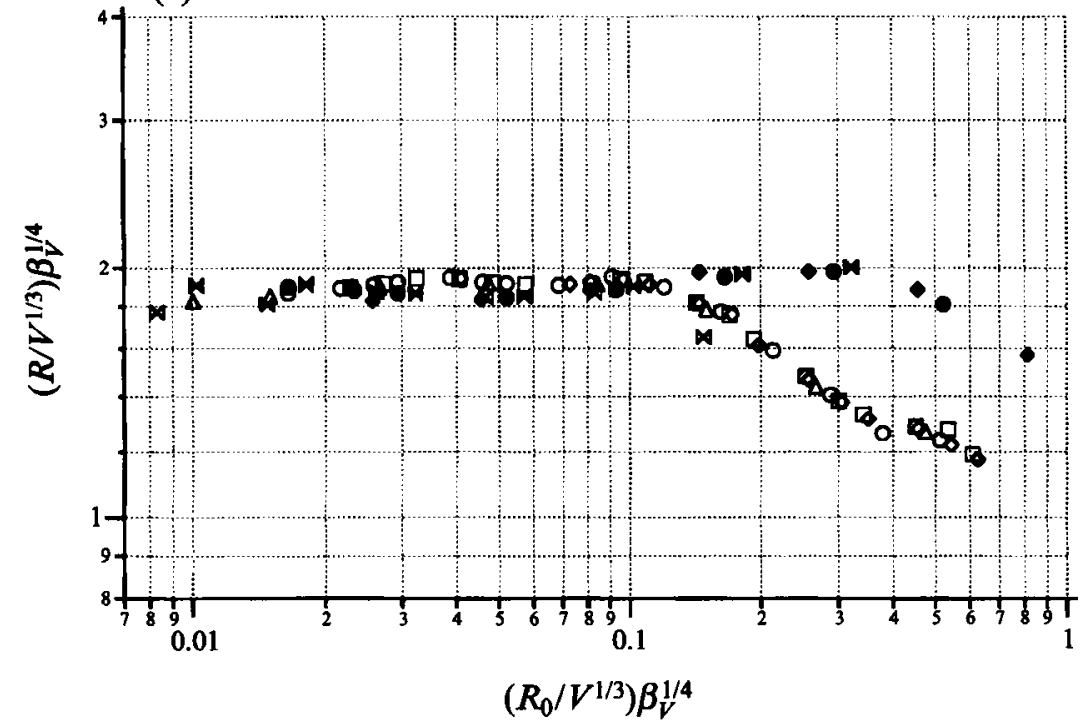

Figure 7. (a) Numerical computation of the extent of deposit $R$ from a fixed-volume current as a function of the modified settling number $\beta_{V}$, and $(b)$ the data replotted to determine the validity of equation (17).

\section{Currents from a constant-flux input}

\subsection{Numerical profiles}

The theoretical height, velocity and concentration profiles for an axisymmetric particle-driven gravity current produced by a constant flux are illustrated in figures 9 and 10. In this simulation the suspension issues at $r=9$ with a dimensionless velocity and height of unity. (The Froude number at the entrance is thus unity.) In order to use our numerical technique, we must have some initial volume of fluid, which for this simulation was assumed to be stationary with $h_{0}=1$ and extending from $r=9$ to $r=10$. Provided the starting volume is reasonably small, the initial 


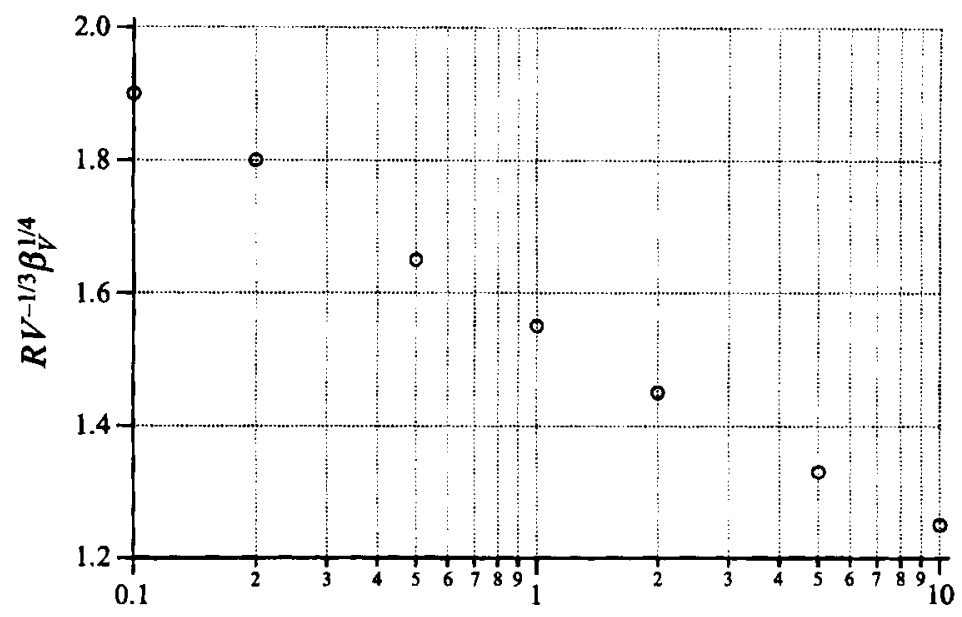

Percentage of particles remaining in suspension

FIGURE 8. The scaled radius $R V^{-1 / 3} \beta_{V}^{1 / 4}$ at which a given percentage of particles remain in suspension in a fixed-volume current, as determined from calculations similar to those of figure 7 . The value of the scaled radius with $0.1 \%$ of particles remaining in suspension defines the constant in (17).

conditions have negligible effect on the subsequent dynamics. The value of the settling number $\beta$ is 0.02 , which is within the range of those in our experiments.

The current can be divided into two regions: an upstream section where the flow is supercritical and a downstream section where the flow is subcritical. As seen in figure 9, the qualitative nature of the profiles is established very quickly after the flow begins and is maintained throughout the simulation (figure 10). In fact the structure of the current is like the self-similar profiles in figure $1(b)$ for a homogeneous current produced by a constant flux even though there is a substantial variation in $\phi$ along the current.

After the current leaves the inlet, its height drops rapidly and its velocity increases to a plateau value. This flow becomes increasingly supercritical as the height of the current decreases. Eventually, this supercritical flow encounters the subcritical flow downstream, and a travelling shock forms between them. We note that the subcritical nature of the downstream flow is determined by the Froude condition at the front of the current.

In the region of supercritical flow, the concentration of particles decreases downstream of the inlet. In this region the concentration is independent of time. Downstream of the shock, however, the concentration is slightly higher than that just upstream. This is due to the greater height and hence lesser rate of settling there. The average concentration of particles in the subcritical region of the flow decreases with increasing time, and this is the cause of the increasing height near the front. At the shock a larger hydrostatic pressure, represented by $\phi h$, is required in the subcritical region of the flow as the conjugate state to the much lower pressure (essentially zero since the particle concentration is very small) in the supercritical region of the flow. As particles settle out, $\phi$ decreases and thus $h$ must increase to maintain the pressure.

\subsection{Experimental results}

Several experiments were performed to measure the radius as a function of time and the areal density of deposit for axisymmetric gravity currents produced by a constant 

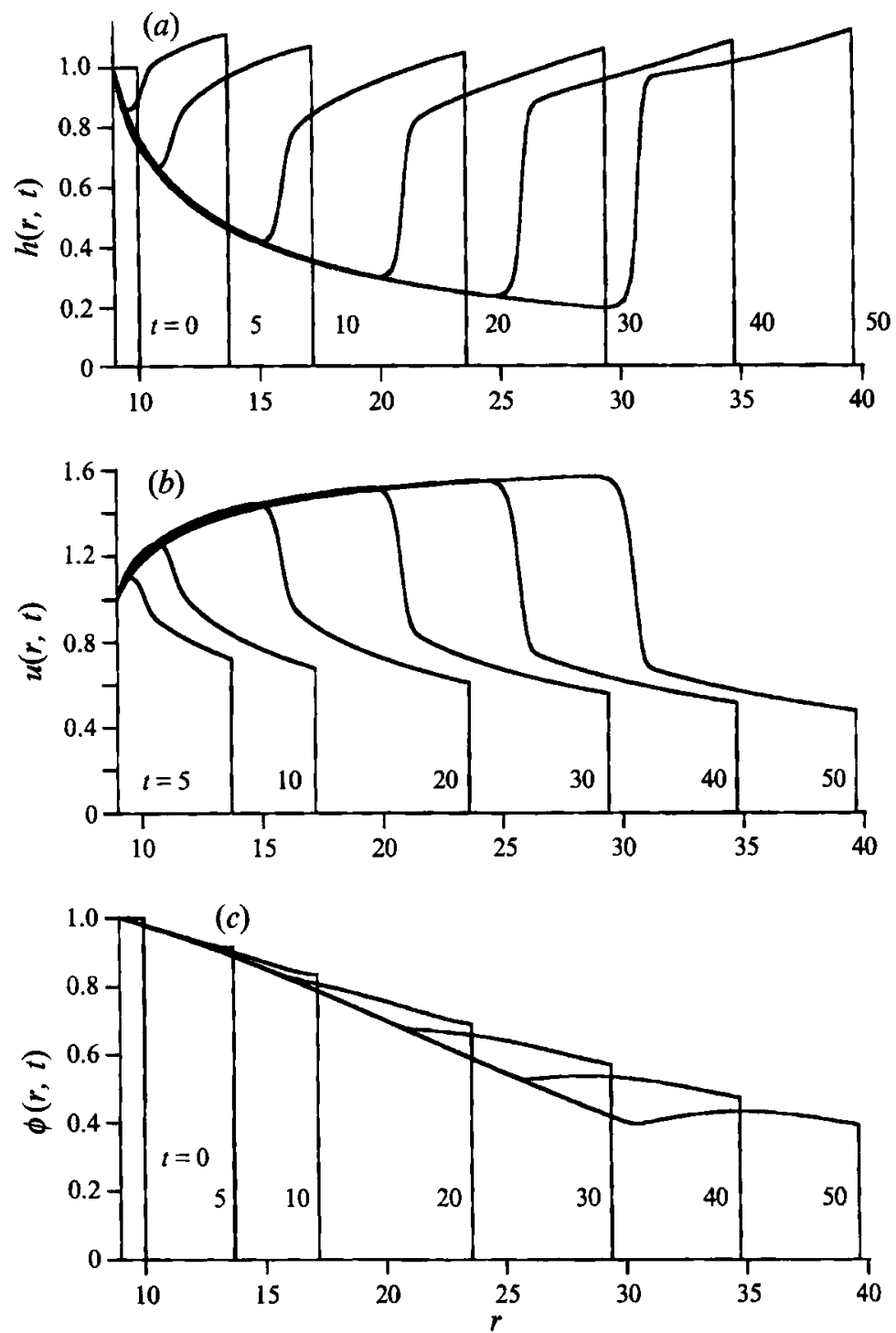

FIGURE 9. The numerically determined profiles of $(a)$ height $h(r, t),(b)$ velocity $u(r, t)$ and $(c)$ concentration $\phi(r, t)$ at early times of a particle-driven gravity current created by the release of a constant flux of suspension. The Froude number at the inlet is unity, and the settling number $\beta=2 \times 10^{-2}$. The successive times are noted by each profile.

flux of suspension. The experiments were performed in the sector tank described earlier and depicted in figure $4(a)$. The rectangular section was modified to include an input system for the suspension, which is illustrated in figure 11. The input system consisted of a vertical tube inserted through a Perspex sheet $23.2 \mathrm{~cm}$ long with foam seals around the edges to fit snugly in the lock. The sheet was placed roughly $3 \mathrm{~cm}$ above the floor of the tank, which was filled to a depth of $14 \mathrm{~cm}$. The presence of the sheet significantly reduces entrainment of the ambient fluid as the suspension is injected into the tank. The suspension was fed to the inlet through the tube, which was connected to a tank containing a well-mixed suspension of silicon carbide particles. 

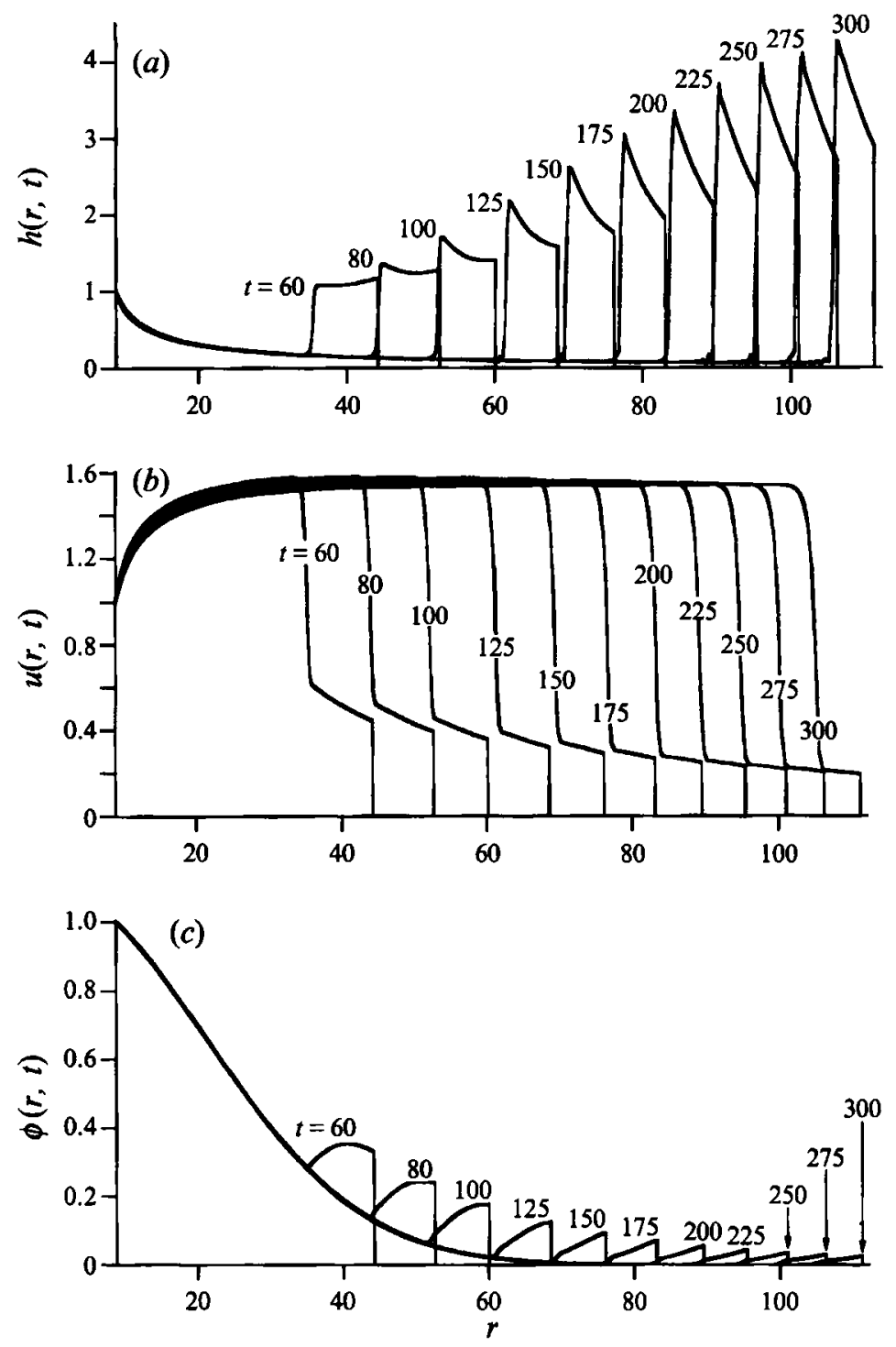

FIGURE 10. The numerically determined profiles at later times for the current described in figure 9.

The suspensions were composed of 37 and $53 \mu$ m-diameter particles $\left(v_{s}=0.17 \mathrm{~cm}\right.$ $\mathrm{s}^{-1}$ and $0.36 \mathrm{~cm} \mathrm{~s}^{-1}$, respectively). The mass of particles in the tank was such that the initial value of the reduced gravity was either 10 or $20 \mathrm{~cm} \mathrm{~s}^{-2}$. Under these conditions the value of $\beta$ ranged from 0.030 to 0.065 . A valve allowed control of the flow rate, which was approximately 75 or $130 \mathrm{~cm}^{3} \mathrm{~s}^{-1}$. The distance between the Perspex plate and the floor was adjusted so that the Froude number at the inlet was near unity. This also significantly reduced entrainment of ambient fluid into the current at the inlet. The duration of the input flux was about $70 \mathrm{~s}$ for each run. The radial position of the current and the density of deposit were measured in the same manner as for the fixed-volume currents. Note that the sediment in suspension when the input ceased was allowed to settle to the floor and was included in the measurement of the density of deposit. 


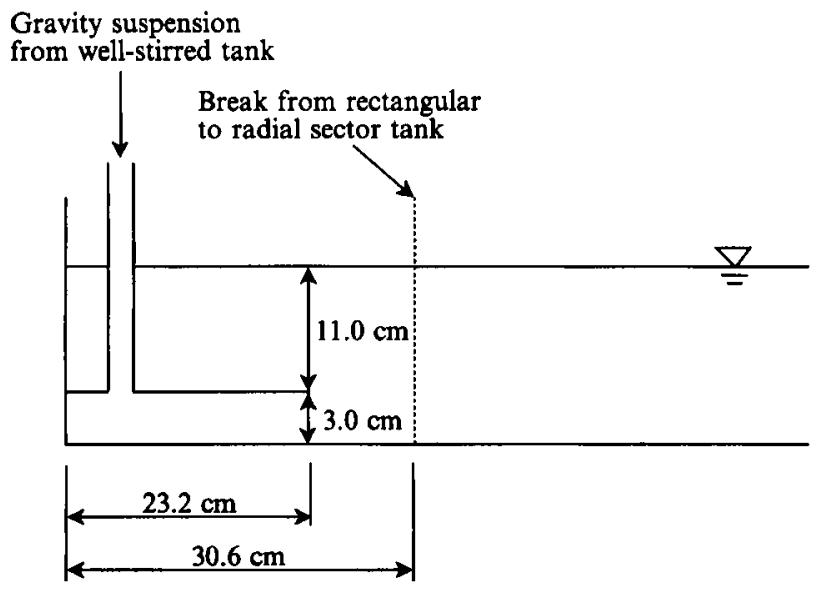

Figure 11. Profile of the input device used to feed suspensions into the rectangular section of the sector tank depicted in figure 4(a) for the experiments with constant flux.

Figure 12 presents the radius as a function of time for the particle-driven gravity currents. The result of an experiment with a saline current is also shown for comparison. Note that the radial distance was measured from the inlet. The radii of the saline current and of the particle-driven current with the same initial reduced gravity were the same for about the first $10 \mathrm{~s}$. The radius of the particle-driven current then increased less rapidly as more particles settled out, decreasing the driving buoyancy force. Generally, at any given time, the radius of a current increased with increasing reduced gravity and with the rate of input.

The numerical predictions of the radial extent of the currents were also computed and are presented in figure 12 . The suspension issuing from the tube quickly spread to fill the $3.8 \mathrm{~cm}$ width of the rectangular section of the tank. So for simulations with the numerical model, it was assumed that the inlet radius was $27.1 \mathrm{~cm}$, which corresponds to the radius of an $8^{\circ}$ sector with an arc of $3.8 \mathrm{~cm}$. This is clearly an approximation to the true geometry, but probably does not significantly affect the results. The radial extent of the saline current is well described by the model for all times, and it increased like $t^{3 / 4}$ as predicted by the similarity solution (equation (10)). The radial extent of the particle-driven gravity currents is well described by the model for most of the duration of the experiment. Towards the end of the experiment, the numerical predictions exceed the measured radial position. At these later times, the front of the current was moving very slowly and the Reynolds number of the current had become sufficiently small that viscous forces became important and further retarded the motion.

Figure 13 presents the density of deposit as a function of radial position for the above particle-driven currents. As we would expect, the density decreased downstream and, for a fixed position, decreased with the initial reduced gravity (or equivalently initial mass loading of particles). Also shown are the numerical predictions of the density of deposits. The general trends of the data are followed and the quantitative agreement is fair. The model predicts more sediment right at the inlet and less sediment slightly downstream than observed. The flow leaving the inlet was rather vigorous due to the nozzle and we suspect that sediment was swept slightly downstream. Note that when computing the density of the deposit from the numerical simulation, any sediment still in suspension above a particular point when the input ceased was 

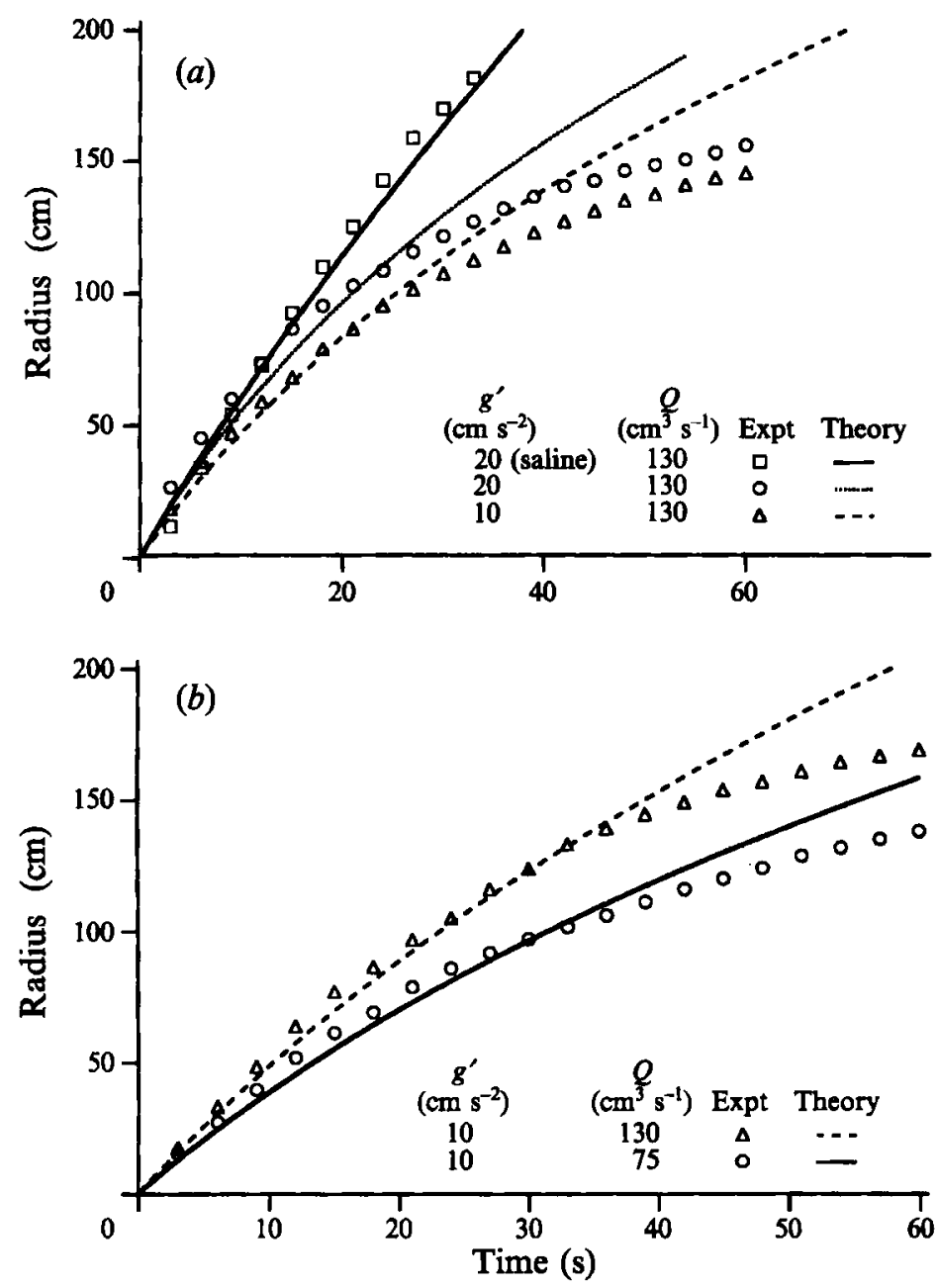

FIGURE 12. The radius as a function of time for axisymmetric gravity currents created by the release of a constant flux of a suspension of $(a) 53$ and $(b) 37 \mu \mathrm{m}$-diameter silicon carbide particles into a tank of fresh water. The symbols are from experimental measurements and the curves are from the numerical solution of the model. The properties of the current are listed in the legend. The radial positions were measured with respect to the virtual origin in figure $4(a)$.

added to the deposit at that point. This only slightly increased the density of deposit near the origin and had no effect far downstream where very little sediment was in suspension.

The extent of deposit produced from the axisymmetric release of a constant flux of suspension at long times can be determined analytically. From the profiles in figure 10 , the concentration of sediment decreases away from the source and is very small near the front. In fact we see that the volume fraction at a given position $r$ becomes constant after the interior shock of the current passes this position. Thus we may assume the flow is at steady state in this region and solve for the volume fraction distribution explicitly. Under these conditions we find that

$$
\phi(r)=\mathrm{e}^{-v_{s} \theta\left(r^{2}-r_{0}^{2}\right) / 2 Q},
$$

where the current is released at the radial position $r_{0}$. This result was derived earlier 

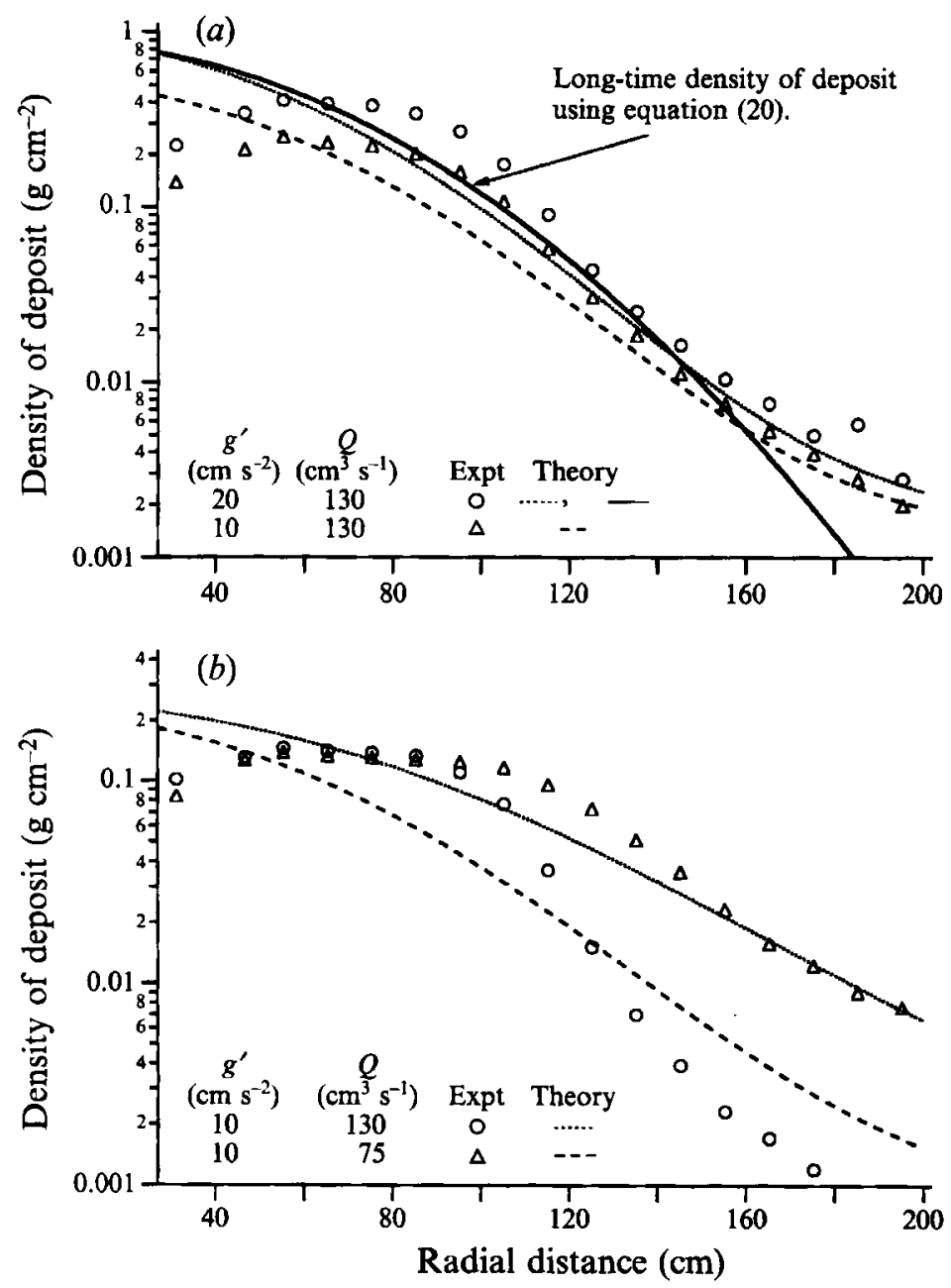

FIGURE 13. The final areal density of the deposit after the flow of axisymmetric particle-driven gravity currents produced by a constant flux of suspension of $(a) 53$ and $(b) 37 \mu$ m-diameter particles. The radial positions were measured with respect to the virtual origin in figure $4(a)$. The duration of the input flux was about $70 \mathrm{~s}$. Also plotted in $(a)$ is the long-time density of deposit predicted using equation (20) for $53 \mu \mathrm{m}$-diameter particles with an initial reduced gravity of $20 \mathrm{~cm} \mathrm{~s}^{-2}$.

by Sparks \& Walker (1977) for the radial distribution of ash raining from a volcanic eruption cloud spreading horizontally along its height of neutral buoyancy.

For locations where the steady-state concentration profile has been established for long times, the density of deposit is predicted by taking the product of $\rho_{p} v_{s} T$ with equation (20), where $T$ is the duration of the flow. The density of deposit for the constant-flux release for $70 \mathrm{~s}$ of a suspension of $53 \mu \mathrm{m}$-diameter particles with an initial reduced gravity of $20 \mathrm{~cm} \mathrm{~s}^{-2}$ is plotted in figure $13(a)$. We observe that it agrees very well with the result from the unsteady numerical simulation for most of the extent of deposit. There is a very slight discrepancy in the mid-range, which is due to the fact that deposition has occurred for less than $70 \mathrm{~s}$ since the current passed over these point after inception of the flow. Very much further downstream, the numerical simulation predicts the density of deposit to be greater than that computed 
from equation (20). The deposition in this region is mostly due to the relatively particle-rich head of the current, which is only captured by the unsteady numerical model.

This discrepancy, however, only occurs where the density of deposit is almost 100 times less than its maximum value. So we may use the steady-state concentration profile to obtain an accurate estimate of the extent of deposition for the axisymmetric release of a constant flux of suspension. Again, defining the extent of the deposit to be where $99.9 \%$ of the sediment has been deposited and using equation (20), we find that $R$ is given by

for $r_{0} \ll R$.

$$
R=3.72\left(\frac{Q}{v_{s} \theta}\right)^{1 / 2}
$$

\section{Conclusions}

We have presented a theoretical model to describe the dynamics and deposition of axisymmetric particle-driven gravity currents created by the release of a fixed volume or constant flux. The dynamics of the current are controlled by a balance between inertial forces in the fluid and buoyancy forces derived from the suspended dense particles. The flow is described by the shallow-water equations, which include the buoyancy effects of the particles. Particles are assumed to be vertically well-mixed due to turbulence, transported along the current by advection and settle out through a viscous sub-layer. A Froude condition at the front of the current is used to model the complex behaviour of the head. No adjustable parameters are needed in the model.

The model matches experimental observations fairly well. For currents of fixed volume, the agreement between the model and experiments for the radial extent is excellent. The agreement was only fair for the deposition pattern, and not as good as we observed for two-dimensional particle-driven gravity currents of fixed volume. This is because a bore is predicted to form during the initial collapse or slumping phase of the flow of the current and it is simply not observed for reasons that are not clear to us.

For currents created by a constant flux of suspension, the radial extent of the current was accurately predicted until viscous forces probably became important. The deposition patterns were also fairly well described. We note, however, that the input conditions were selected so that the Froude number at the inlet was near unity to decrease the effects of entrainment of ambient fluid. For supercritical inlet conditions there was a significant amount of entrainment, which is not included in our model. In these cases, the model and experiments would not agree very well unless the model were augmented by entrainment of ambient fluid at the upper surface.

Although the model has some shortcomings, it is computationally very fast, gives reasonable results and has no adjustable parameters. In addition the model provides a framework and rapid means of conducting numerical experiments, which can be used, for example, to produce simple estimates for the extent of the deposit from a particle-driven gravity current as we did here. The fact that the model has no adjustable parameters is especially important when modelling a completely new system, since it is not necessary to guess the values of the parameters. These characteristics make our approach ideal for modelling other aspects of particle-driven gravity currents. In particular, there is a great deal of interest in determining the deposition pattern from currents created by sediment-laden outflows from rivers over 
topography. These deposits, such as those in the Gulf of Mexico and off the coast of Southern California, have become valuable oil reservoirs, and knowledge of their origin is useful for exploration and exploitation of this resource. We plan to use our model to develop an understanding of their origin.

We are grateful to R. E. Britter, W. B. Dade, R. C. Kerr, L. W. Lake, and H. M. Pantin for helpful comments on a previous version of this manuscript. We also thank G. F. Lane-Serff for encouraging us to make the comparison with the box model in the Appendix. This work was partially supported by NERC.

\section{Appendix. A box model for axisymmetric particle-driven gravity currents}

We present here a simple box model for comparison with our more detailed calculation of the dynamics and deposition of an axisymmetric particle-driven gravity current. Such models have been derived previously by Huppert \& Simpson (1980) for homogeneous gravity currents and by Dade \& Huppert (1994) for two-dimensional particle-driven gravity currents. The derivation below follows closely that of Dade \& Huppert (1995).

We approximate the gravity current as a cylinder whose radius $r(t)$ and uniform height $h(t)$ evolve as the current propagates. The particles in the current are assumed to be completely well-mixed so that the volume fraction $\phi$ is uniform both horizontally and vertically throughout the current. The velocity of the front of the current obeys the Froude condition of equation (5)

$$
\mathrm{d} r / \mathrm{d} t=\operatorname{Fr}\left(g_{0}^{\prime} \phi h\right)^{1 / 2},
$$

where $g_{0}^{\prime}=\left(\rho_{p}-\rho_{a}\right) \phi_{0} / \rho_{a}$ and $\phi$ is the volume fraction of particles scaled with the initial volume fraction $\phi_{0}$. This equation represents the balance of inertial and buoyancy forces in the current. The equation describing the conservation of particles is

$$
\mathrm{d} \phi / \mathrm{d} t=-v_{s} \frac{\phi}{h} .
$$

Finally, conservation of mass requires that $\pi r^{2} h=V$, where $V$ is the volume of the current, which remains constant. We assume here that the ambient fluid is much deeper than the current and so $F r=1.19$. Equations (A1) and (A2) can be combined and solved to show that for an instantaneous point release

$$
(r / R)^{4}+\phi^{1 / 2}=1
$$

where

$$
R=\left(64 F r^{2} / \pi^{3}\right)^{1 / 8}\left(g_{0}^{\prime} V^{3} / v_{s}^{2}\right)^{1 / 8}
$$

is the maximum distance travelled by the current and hence also the extent of the deposit. Note from (A4) that we would compute the proportionality constant in equation (16) to be $\left(8 \mathrm{Fr} / \pi^{3 / 2}\right)^{1 / 4}=1.1$, which is about $60 \%$ of the value 1.9 predicted from the full shallow-water calculation.

Using (A3), we can integrate (A1) and (A2) directly and, after a bit of algebra, we find that the radius of the current and the volume fraction of suspended particles as functions of time are given by

$$
r=R \tanh ^{1 / 2}(t / \tau)
$$


and

$$
\phi=\operatorname{sech}^{4}(t / \tau)
$$

where

$$
\tau=\left(4 / F r^{2} \pi\right)^{1 / 4}\left(V / v_{s}^{2} g_{0}^{\prime}\right)^{1 / 4}
$$

The density of deposit $D(r)$ is the flux of sediment integrated from the time $t^{\circ}(r)$ when the current reaches position $r$ to $t=\infty$, or

$$
D(r)=\rho_{p} v_{s} \phi_{0} \int_{r^{-(r)}}^{\infty} \phi(t) \mathrm{d} t,
$$

where $t^{*}(r)=\tau \operatorname{arctanh}\left[(r / R)^{2}\right]$. Using (A6), we can compute this integral exactly and show that the density of deposit is given by

$$
D(r)=\frac{2}{3} \rho_{p} \phi_{0} \tau\left(1-\frac{3}{2}(r / R)^{2}+\frac{1}{2}(r / R)^{6}\right)
$$

The radius of the current as given by (A5) is plotted in figure 14(a) for comparison with the shallow-water model and with the experimental measurements for currents composed of $23 \mu \mathrm{m}$-diameter particles. The box model predicts the radius as a function of time reasonably accurately for the larger initial volume fractions of particles. In the experiment the ambient fluid is in fact comparable in depth to that of the initial current and the assumption of a constant Froude number is not correct during the initial slumping stage. The smaller the initial concentration of particles, the longer the current remains in the slumping regime, and hence the larger the error. This could be corrected by adopting a variable Froude number, though it would make the model more complicated than warranted. The density of deposit predicted from the straightforward box model is illustrated in figure $14(b)$. The box model does not predict the experimentally observed maximum in the deposit near the origin. From the figure we conclude that the box model is no better than the shallow-water model in predicting the density of deposit.

When first trying to model their experiments on two-dimensional gravity currents, BHL investigated a two-dimensional box model. However, they found that the length as a function of time and the density of deposit could not be described accurately by such an approach. As mentioned earlier in the main text, the spatial and temporal evolution of the depth of the current are critical in determining the density of deposit accurately and, for two-dimensional currents, the two-layer shallow-water equations, which correctly predict the detailed structure of the current, were necessary to model the deposition.

The box model presented here has the advantage of producing simple analytical formulae for the radius as a function of time and for the density of deposit. While the box model also produces order-of-magnitude estimates for the extent of the deposit that are dimensionally equivalent to those from the shallow-water equations, it neither explains the absence of the internal shock during the slumping phase nor predicts the density of the deposit more accurately than the shallow-water model presented here. Indeed, the box model does not attempt to describe any of the internal structure predicted by the shallow-water model, but concentrates only on the global mass, momentum and particle balances. The box model also has the disadvantage of not being extendable to account for the effect of topographical variations or variations in resuspension that might occur along the length of the current. 

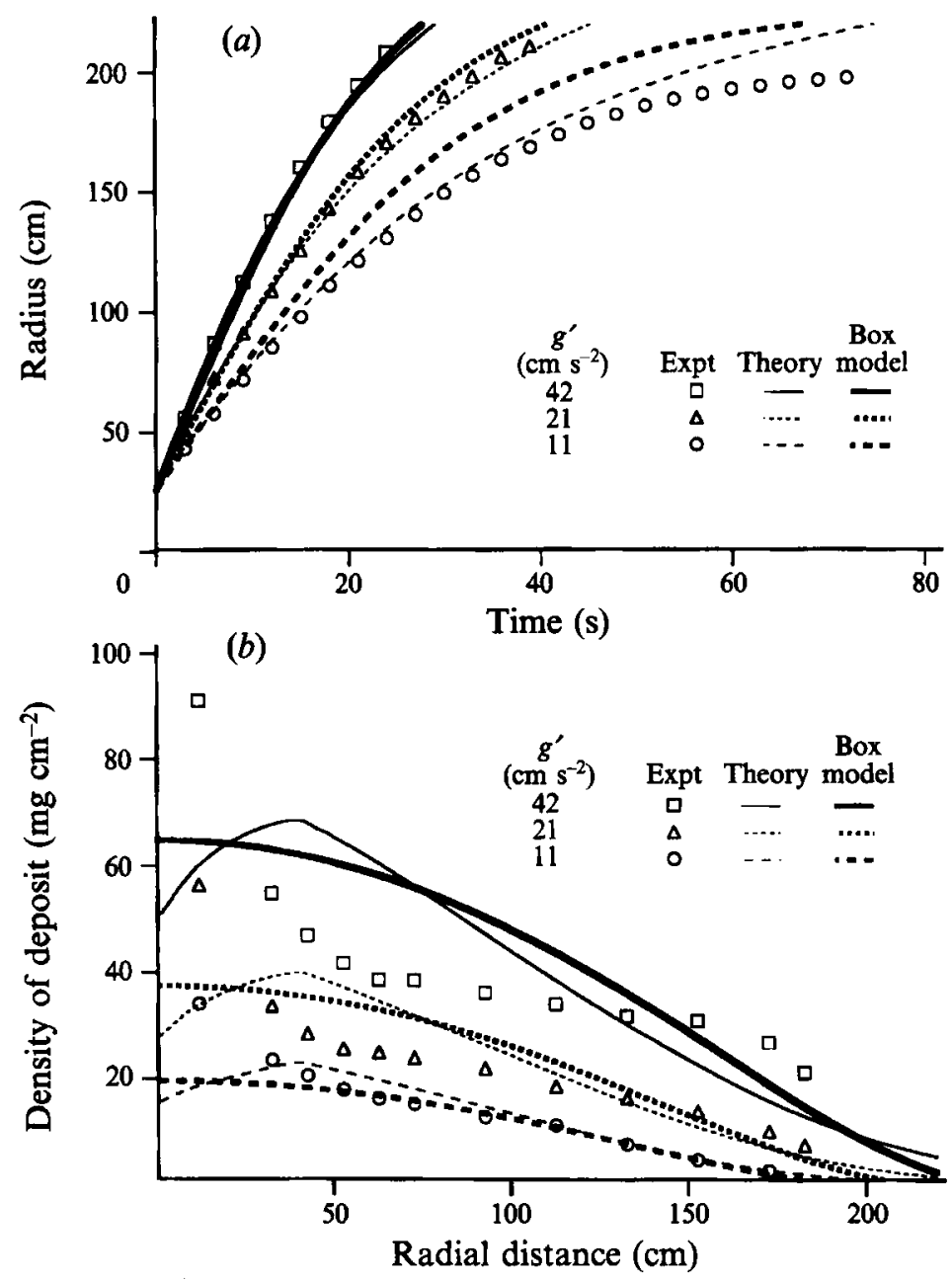

FIGURE 14. The radius as a function of time (a) and the density of deposit (b) for axisymmetric gravity currents composed of suspensions of $23 \mu \mathrm{m}$-diameter silicon carbide particles released into a tank of fresh water. The radial positions were measured with respect to the virtual origin in figure 4(a). The symbols are from experimental measurements, the thinner curves are from the numerical solution of the theoretical model presented in the main text and the thicker curves are from solution to the box model presented in the Appendix. The properties of the current are listed in the legend.

\section{REFERENCES}

Benjamin, T. B. 1968 Gravity currents and related phenomena. J. Fluid Mech. 88, 223-240.

BonNeCAZe, R. T., Huppert, H. E. \& Lister, J. R. 1993 Particle-driven gravity currents. J. Fluid Mech. 250, 339-369 (referred to herin as BHL).

CHEN, J. C. 1980 Studies on gravitational spreading currents. PhD thesis, California Institute of Technology.

DADE, W. B. \& HUPPERT, H. E. 1994 Predicting the geometry of deep-sea channelized turbidites. Geology 22, 645-648.

DADE, W. B. \& HUPPERT, H. E. 1995 Runout and fine-sediment deposits of axisymmetric turbidity currents. J. Geophys. Res. (Oceans), sub judice.

EINSTEIN, H. A. 1968 Deposition of suspended particles in a gravel bed. J. Hydraul. Div. ASCE 94, 1197-1205. 
Gratton, J. \& Vigo, C. 1994 Self-similar gravity currents with variable inflow revisited: plane currents. J. Fluid Mech. 258, 77-104.

Grundy, R. E. \& RotTMAN, J. W. 1986 Self-similar solutions of the shallow-water equations representing gravity currents with variable inflow. J. Fluid Mech. 169, 337-351.

HAY, A.E. 1987 Turbidity currents and submarine channel formation in Rupert Inlet, British Columbia, 1. Surge observations. J. Geophys. Res. 92, 2875-2881.

Hoult, D. P. 1972 Oil spreading on the sea. Ann. Rev. Fluid Mech. 4, 341-368.

HUPPERT, H. E. 1982 The propagation of two-dimensional and axisymmetric viscous gravity currents over a rigid horizontal surface. J. Fluid Mech. 121, 43-58.

Huppert, H. E. \& Simpson, J. E. 1980 The slumping of gravity currents. J. Fluid Mech. 90, 785-799.

InMaN, D.L., Nordstrom, C.E. \& FuICK, R.E. 1976 Currents in submarine canyons: an air-sea-land interaction. Ann. Rev. Fluid Mech. 8, 275-3109.

KÁRmÁN, T. von 1940 The engineer grapples with nonlinear problems. Bull. Am. Math. Soc. 46, 615-683.

MaRTIN, D. \& NoKES, R. 1988 Crystal settling in a vigorously convecting magma chamber. Nature 332, 534-536.

MARTIN, D. \& NokES, R. 1989 A fluid-dynamical study of crystal settling in convecting magmas. J. Petrol. 30, 1471-1500.

MCCAVE, I.N. 1970 Deposition of fine grained suspended sediment from tidal currents. J. Geophys. Res. 75, 4151-4159.

NoRmaRK, W.R. \& Dickson, F.H. 1976 Man-made turbidity currents in Lake Superior. Sedimentology 23, 815-831.

Perrodon, A. 1985 Dynamics of oil and gas accumulation. Bulletin des Centres de Recherches Exploration-Production, Elf-Aquitaine, Pau, France, pp. 98-105.

Simpson, J. E. 1987 Gravity Currents in the Environment and the Laboratory. Ellis Horwood.

Sparks, R. S. J., Bonnecaze, R. T., Huppert, H. E., Lister, J. R., Mader, H. \& Phillips, J. 1993 Sediment-laden gravity currents with reversing buoyancy. Earth Planet. Sci. Lett. 114, 243-257

SPARKS, R.S.J. \& WALKER, G.P.L. 1977 The significance of vitric-enriched air-fall ashes associated with crystal-enriched ignimbrites. J. Volcanol. Geotherm. Res. 2, 329-341.

Whitham, G.B. 1974 Linear and Nonlinear Waves, p. 456. John Wiley \& Sons. 